\title{
GASPHOT: a tool for Galaxy Automatic Surface PHOTometry
}

\author{
E. Pignatelli ${ }^{1}$, G. Fasano ${ }^{1}$, and P. Cassata ${ }^{2}$
}

\author{
1 Osservatorio Astronomico di Padova, Vicolo dell'Osservatorio 5, 35122 Padova, Italy \\ e-mail: pignatelli@pd.astro.it \\ 2 Dipartimento di Astronomia, Università di Padova, Vicolo dell'Osservatorio 3, 35122 Padova, Italy
}

Received 21 July 2004 / Accepted 28 July 2005

ABSTRACT

In this paper we present a new tool for automatic, blind surface photometry of galaxies in deep and/or wide fields. The tool (GASPHOT) favors the robustness of results with respect to the details of galaxy modeling. To this aim, a single Sersic-law for the models and a hybrid 1D/2D approach for the best-fitting algorithm were adopted. GASPHOT is heavily based on SExtractor and provides luminosity, position angle and ellipticity profiles of galaxies extracted from CCD frames, as well as total magnitudes, half-light radii, and Sersic indices. These global parameters were obtained by simultaneously fitting the major and minor axis light growth curves of galaxies with a 2D flattened Sersiclaw, convolved by the appropriate, space-varying PSF, which was previously evaluated by the tool itself. We tested GASPHOT on more than 15000 simulated and real galaxies, obtaining robust upper limits for the errors of the global parameters of galaxies, even for non-Sersic profiles and blended objects. The GASPHOT performance was also compared with those of two popular alternative tools for a single component, Sersic law: GALFIT (Peng et al. 2002) and GIM2D (Marleau \& Simard 1998). When dealing with ideal, isolated, and adeguately large galaxies, the three packages were all found to provide satisfactory results, apart from a definite bias in the GIM2D magnitudes. On the other hand, GASPHOT proved to be more robust than GALFIT and GIM2D for simulated, blended objects and for real galaxies. Moreover, at variance with GASPHOT and GALFIT, the quality (bias and scatter) of the fits provided by GIM2D rapidly declines as the galaxy size decreases.

Key words. methods: data analysis - galaxies: photometry - galaxies: fundamental parameters

\section{Introduction}

In the past few years, the introduction of new generation of wide-field CCD imagers for astronomy has yielded a huge amount of data appropriate for studying the structure, formation, and evolution of galaxies. In particular, ground-based wide-field instruments like CFHT-12K, AAT-WFI, MPG/ESOWFI, and INT-WFC are providing large databases for morphological and evolutionary studies of galaxies at low and intermediate redshifts (McMahon et al. 2001; Andreon \& Cuillandre 2002; Durret et al. 2002; Pracy et al. 2003; Fasano et al. 2003; Hudson et al. 2003; Pimbblet \& Drinkwater 2004), while highresolution, very deep HST(WFPC2/ACS) observations of distant galaxies are giving us a glimpse at their early evolutionary phases (Blakeslee et al. 2004; Ravindranath et al. 2004; Thompson 2004; Rix et al. 2004; Cimatti et al. 2004; Cassata et al. 2005). This kind of data is invaluable for the huge information it contains. However, it also introduces new problems, mainly related to the unprecedented amounts of data that must be managed, which we are still not able to fully exploit because of the inadequacy of the tools used to extract the physical information.

The Source Extractor package (SExtractor; Bertin \& Arnouts 1996) has brilliantly solved this issue, as far as the aperture photometry of extended sources is concerned, thereby providing a number of different photometric and geometric parameters for each galaxy in a given CCD image. However, typical tasks for extragalactic researchers include morphological classification of galaxies and measurement of their global, photometric, and structural parameters (total magnitude, half-light radius, Sersic index, etc.). The first task has been performed, so far, by experienced human classifiers, usually accomplished for no more than a few hundred objects. The second task implies extracting the radial luminosity and geometrical profiles of galaxies and fitting suitable analytic laws to them. Obviously, to accomplish such a task on wide/deep-field images, one cannot rely on the standard surface photometry tools like IRAF-ELLIPSE (Jedrzejewski 1987) or AIAP (Fasano 1994; Fasano et al. 1996), which produce ellipse-fitting-based (1D) luminosity and geometrical profiles and are optimized to be used on single objects. New tools that are able to efficiently handle hundreds, or even thousands of galaxies per run are clearly needed. Recently, two packages for best-fitting galaxy images with 2D multi-component models were developed and applied to relatively sizeable galaxy samples: GIM2D (Marleau $\&$ Simard 1998) and GALFIT (Peng et al. 2002). However, the robustness of these automatic surface photometry tools for real galaxies (often far from following the models) is not quantifiable enough. In particular, since the $2 \mathrm{D}$ best-fitting approach is likely to be sensitive to the features of real galaxies more than 
the $1 \mathrm{D}$ approach is, its blind application could produce unrealistic results for a fraction of the galaxy sample.

Two of us (EP and GF) are deeply involved in a long-term project (WINGS, Fasano et al. 2003) exploiting wide-field instrumentation to build up huge photometric $(B, V, J, K)$ and spectroscopic databases of galaxies in a complete sample of $78 \mathrm{X}$-Ray selected clusters of the local Universe $(0.04<z<$ 0.07). Within the framework of the WINGS project, it has been planned to produce detailed surface photometry and morphological classification for about 500 objects per cluster, a total of $\sim 40.000$ galaxies. The WINGS project clearly requires fully automatic tools for both surface photometry and morphological classification of galaxies.

The last issue (morphological classification) remains largely unsolved by all (both 1D and 2D) available surface photometry tools, independent of their degree of interactivity. As a matter of fact, there are two parameters derived from the galaxy surface brightness distribution that could indicate something about morphology: the Sersic's index $n$ (Sersic 1968; Ciotti 1991 ) and the bulge-to-disk ratio (more correctly, the $L_{R^{1 / 4}} / L_{\exp }$ best-fitting parameter). Unfortunately, while both quantities appear to be correlated with the morphological classification based on human-eye inspection, the scatter in this correlation is still large enough to prevent its use for any morphological classification (Abraham et al. 1996; Simien \& de Vaucouleurs 1986; Caon et al. 1993).

In the past few years, different authors have proposed different quantitative classification schemes. Among them, the most popular is the so-called CAS parameter set, including the Concentration (C), Asymmetry (A) and Clumpiness (S) indices (Abraham et al. 1996; Conselice et al. 2000; Conselice 2003), which have also been combined with colors and a power spectrum in Fourier space (Takamiya 1999; Odewahn et al. 2002). Such quantitative morphological indicators, however, are quite rough, and not comparable with visual classification, as far as both the accuracy and the robustness are concerned. In a forthcoming paper (Fasano et al. 2005), the Sersic index $n$ will be used in combination with the CAS parameters set and with a number of additional ad hoc indicators to give robust automatic estimates of the morphological classification of galaxies.

In this paper we present a new surface photometry package named GASPHOT (Galaxy Automatic Surface PHOtometry Tool), which substantially reduces the amount of interaction for the user and provides the relevant global parameters for the hundreds of galaxies typically found in wide/deep-field images. At variance with GALFIT and GIM2D, we fit the photometric profiles in a way which is not completely $2 \mathrm{D}$, but more similar to the ellipse-fitting algorithm usually adopted by the interactive (single object) tools. Using this procedure, we find that there is no loss of precision with respect to the $2 \mathrm{D}$ approach, while there is a gain in robustness of the results. The resulting parameters include total magnitude, effective radius, Sersic index $n$, and axial ratio. GASPHOT is closely based on SExtractor, which is used to produce the photometric profiles of all objects in the input catalog. We devoted a good deal of effort to checking the biases and the robustness of the results by applying the tool to large datasets of simulated and real galaxies. Besides providing realistic errors of the computed global parameters for each galaxy, this, more importantly, led us to answer the crucial question concerning the reliability of the results when the tool is applied to real galaxies. It is worth noticing that, besides the extensive use of GASPHOT in the framework of the WINGS project (Fasano et al. 2003), the tool has already been used successfully to produce several other scientific results (Cimatti et al. 2003, 2004; Cassata et al. 2005).

After outlining the strategy of GASPHOT and its structure (Sects. 2 and 3), we show the results of the extensive testing done on simulated galaxies in Sect. 4, including non-Sersic profiles and blended objects and giving robust upper limits for the error bars of the different parameters. In Sect. 5 we applied the tool to real galaxies and checked the photometric values obtained against previously published results for the same objects. Finally, in Sect. 6 we compare our results with those from both GALFIT and GIM2D, and in Sect. 7 we summarize the results.

\section{The strategy of GASPHOT}

The primary goal of GASPHOT is to extract, in a fully automatic and blind mode, reliable estimates of the main photometric and structural parameters of large samples of galaxies from wide/deep-field images. The tool must be reliable enough to provide reasonable results even under critical conditions (small and/or low surface brightness and/or blended objects) and thereby to eliminate the need to guess the model parameters. Matching these kinds of requirements calls for drastic choices concerning both the allowed models and the algorithm to be used in the best-fitting procedure. In both cases we have to find an acceptable compromise between the tendency to describe the galaxy as fully as possible and the strong constraints rising from the requirements of blindness and robustness that we want to attain.

As for the choice of the models adopted to represent the galaxy profiles, it is worth considering that the typical target galaxies that GASPHOT will deal with (WINGS-like images) are usually not spatially resolved enough to demand (or even to allow) a detailed multi-component description. Therefore, we decided once and for all to keep just a single Sersic law component to represent the light distribution of galaxies. We are aware that this is quite a drastic choice. However, the Sersic law is flexible enough to give a fair representation of most galaxy profiles, being at the same time relatively simple (small amount of parameters) and easy to handle by both the convolution and best-fitting algorithms. These features match the robustness requirement, while not compromising (as we will show) the accuracy of the results as far as the global structural parameters of galaxies are concerned (i.e. total intensity, effective radius, ellipticity, position angle). In Sect. 4.2 we show that the Sersic index $n$ also correlates loosely with the bulge/disk ratio.

The strategy we have chosen in order to approach the bestfitting problem is somehow a hybrid between the 1D (equivalent luminosity profile fitting) and 2D (full image fitting) approaches. The $1 \mathrm{D}$ technique has the advantage of being less sensitive to the peculiar features of real galaxies, since the parameters defining each single isophote (coordinates of the center, area, flattening, and position angle) are averaged over a large number of pixels and can also be derived for very 


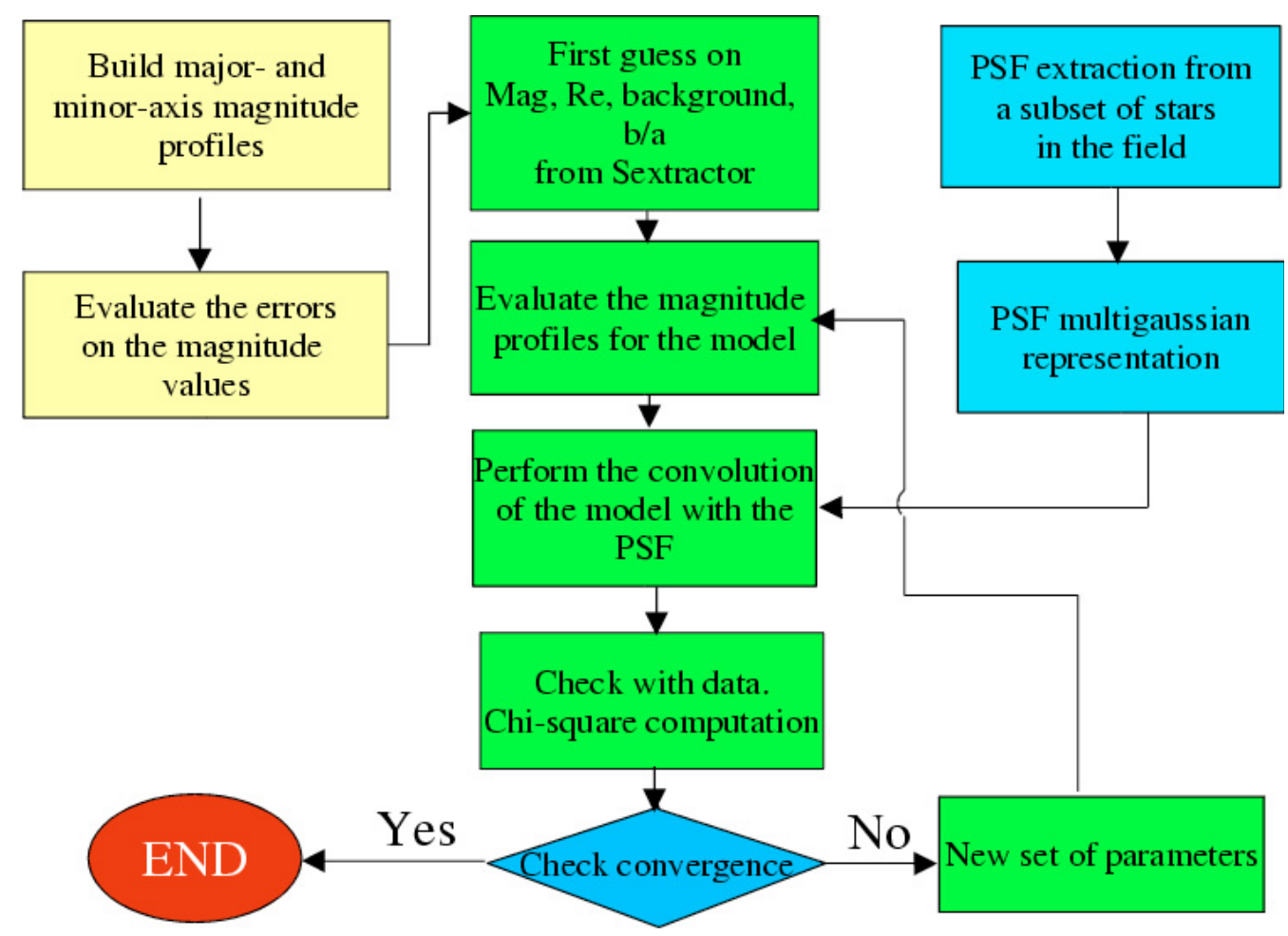

Fig. 1. Flow-chart of the basic structure of GASPHOT.

irregular isophotes. Thus, it is capable of also handling the outer regions of galaxies and the presence of either oddness or asymmetry of the galaxy shape. On the other hand, the $1 \mathrm{D}$ technique is unable to model either the inner (seeing-affected) regions of flattened galaxies or possible misalignments between different galaxy components. Instead, the $2 \mathrm{D}$ approach is fully appropriate to such modeling, but is more affected by any irregularity in the galaxy's shape. Roughly speaking, the 1D approach is more robust, since it provides reasonable results even in critical situations, while the $2 \mathrm{D}$ approach is suitable for accurate, detailed modeling of well-sampled objects, even for multicomponent structures and in the very inner region of galaxies.

GASPHOT tries to exploit the robustness of the 1D fitting technique, keeping at the same time the capability of dealing with PSF convolution in the innermost regions typical of the $2 \mathrm{D}$ approach. In practice, the tool performs a simultaneous $\chi^{2}$ best-fit of the major and minor axis growth light curves of galaxies, by properly convolving the corresponding profiles of the model.

\section{The structure of GASPHOT}

The structure of the tool is illustrated by the flow-chart in Fig. 1, and can be summarized by the following steps:

1. Creating (or self-providing) two reference catalogs from the input image including: (i) galaxies for which we seek surface photometry; and (ii) stars suitable for the analysis of the Point Spread Function (PSF);

2. Extracting isophotal and aperture profiles of galaxies and stars included in the two reference catalogs;
3. Modeling a space-varying PSF from the luminosity profiles of stars;

4. Evaluating the global photometric parameters of galaxies by fitting a Sersic law (convolved by the appropriate, local PSF) to their growth light curves, derived along both the major and the minor axes.

\subsection{The reference catalogs}

In its standard operating mode, GASPHOT will run a preliminary step in order to build the reference catalogs for stars and galaxies directly from the image, according to the constraints indicated by the user.

The user may also optionally provide his own catalogs for stars and galaxies. In this case, GASPHOT will run the same preliminary step, but using the user-provided reference catalog for association. Obviously, one can interactively clean the catalogs before going on with the next steps. The user catalogs must just contain the precise coordinates - in pixels - of the galaxies and/or stars to be selected.

\subsection{The catalogs of photometric profiles}

We exploit the capabilities of SExtractor in order to simultaneously produce a complete set of isophotes for galaxies and stars in their respective reference catalogs.

The catalogs of photometric profiles for galaxies are extracted running several times Sextractor (with the input reference catalogs used for association) on both the input and the segmentation image, by cycling on the isophotal threshold with a given step of surface brightness (usually a few hundredths 
of magnitude). Each run slices the input image and produces the isophotes corresponding to a given value of the surface brightness for the whole galaxy sample. For each isophote, SExtractor provides the coordinates of the center, the isophotal area, the integrated magnitude within that area, the position angle $(\theta)$, the axial ratio $(b / a)$, and a numerical code which flags possible problems affecting the isophote (blending, saturation, closeness to the image boundary, etc.). Note that $\theta$ and $b / a$ are not luminosity-weighted values (those provided by SExtractor on the input image), but "true" isophotal quantities, computed on the segmentation image with FILTERing off. Additional SExtractor runs provide photometry over a set of Kron-like elliptical apertures with fixed (Kron) axial ratio beyond the lowest threshold, which is set by default to $2.5 \sigma$ of the background noise (see Sect. 3.4). In this way, we obtain both the isophotal and aperture magnitudes that we combine in a single catalog, including surface brightness, ellipticity, and position angle profiles as a function of the isophotal semi-major axis. Isophotes having flag greater than 1 (see SExtractor user's guide) and elliptical apertures with positive flag will not contribute to the $\chi^{2}$ in the best-fitting procedure.

It is important to stress that, once the basic SExtractor parameters are set in a configuration file, the program produces luminosity and geometrical profiles of the whole sample of galaxies in fully automatic mode. With this procedure we can take full advantage of the built-in deblending algorithm of SExtractor to separate nearby objects.

\subsection{Modeling the PSF profile}

At the same time, in order to obtain a careful representation of the PSF through the field, GASPHOT extracts the equivalent luminosity profiles of the stars in the image. "Good stars" are defined as those objects with a SExtractor Star/Galaxy parameter greater than 0.9 and an axial ratio greater than some user-defined value (usually 0.9 ), but also that are not blended or saturated and are far enough from the border of the image.

GASPHOT automatically extracts the profiles of the stars, models the variation of the FWHM through the field with a user-defined degree 2D polynomial, and combines the PSF profiles, after having re-scaled them according to the spacevarying model obtained previously. Finally, a multi-Gaussian function is used to perform the $\chi^{2}$ best-fitting of the average PSF profile. The procedure adopts an iterative rejection algorithm in order to remove possible outliers and non-stellar objects from the fit. Figure 2 illustrates the PSF that results from the INT wide-field imaging of the galaxy cluster Abell 2256 obtained in the framework of the WINGS project. The multiGaussian, space-varying PSF profile will be used to properly convolve the Sersic law in the fitting procedure of the observed luminosity profiles (major/minor axis) of each galaxy. Since the number of Gaussians has a strong impact on the computing time needed for the best-fitting, GASPHOT will try an acceptable fit of the PSF with as few Gaussians as possible.

We note that the profiles of stars and galaxies are extracted in a fully consistent way. This means that possible distortions of the "real" galaxy profiles induced by the extraction algorithm

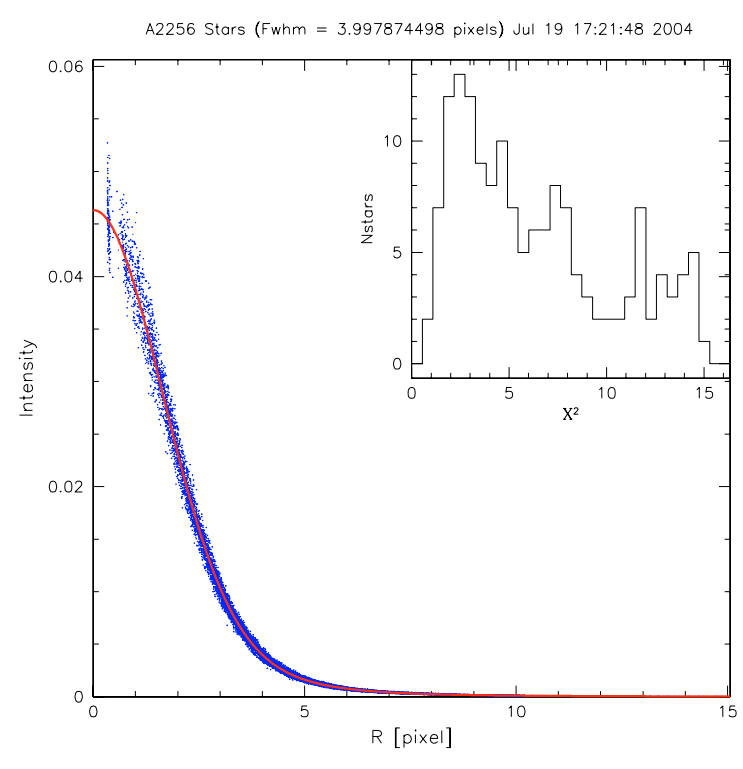

Fig. 2. Point spread function extracted by GASPHOT (see text) for an INT image of the cluster Abell 2256, using more than 500 stars in the field. The normalized and re-scaled profiles of the different stars are shown together with the best fit superimposed on them. The rightupper panel shows the best-fit reduced $\chi^{2}$ distribution for the stars, which is used to automatically remove outliers from the fit.

will be also present as a "fake" component of the PSF profile and will be then, at least partially, corrected during the bestfitting procedure, when convolving the theoretical profiles.

In a few cases (e.g. when the image is not properly sampled and too few stars are available), it is possible that the user might be not completely satisfied with the results of the PSF modeling. It is, however, always possible for the user to provide his own multi-Gaussian modeling of the PSF, as well as the polynomial representing the variation of the FWHM through the image, before proceeding with the next steps.

\subsection{Fitting the luminosity profiles of galaxies}

Once we have produced the analytical (multi-Gaussian), spacevarying representation of the PSF profile and the catalog of the photometric profiles of all target galaxies, we can proceed to fitting these profiles and retrieving the global photometric and structural parameters of galaxies.

As explained in Sect. 2, we assume that all galaxies have surface brightness profiles that can be represented by a Sersic law (Ciotti 1991; Trujillo et al. 2001):

$\ln \left[I(R)-I_{\mathrm{bkg}}\right]=\ln \left(I_{0}\right)-k\left(\frac{R}{R_{\mathrm{e}}}\right)^{n}$

where the free parameters are: the Sersic index $n$, the effective radius $R_{\mathrm{e}}$, the central surface brightness $I_{0}$ (or, equivalently, the total luminosity), and the background sky level $I_{\mathrm{bkg}}$. The de Vaucouleurs law corresponds to a Sersic index $n=4$ and a pure exponential law to $n=1$. The parameter $k$ is univocally defined as a function of $n$ with the condition that $R_{\mathrm{e}}$ be the radius enclosing half of the total luminosity of the object. 

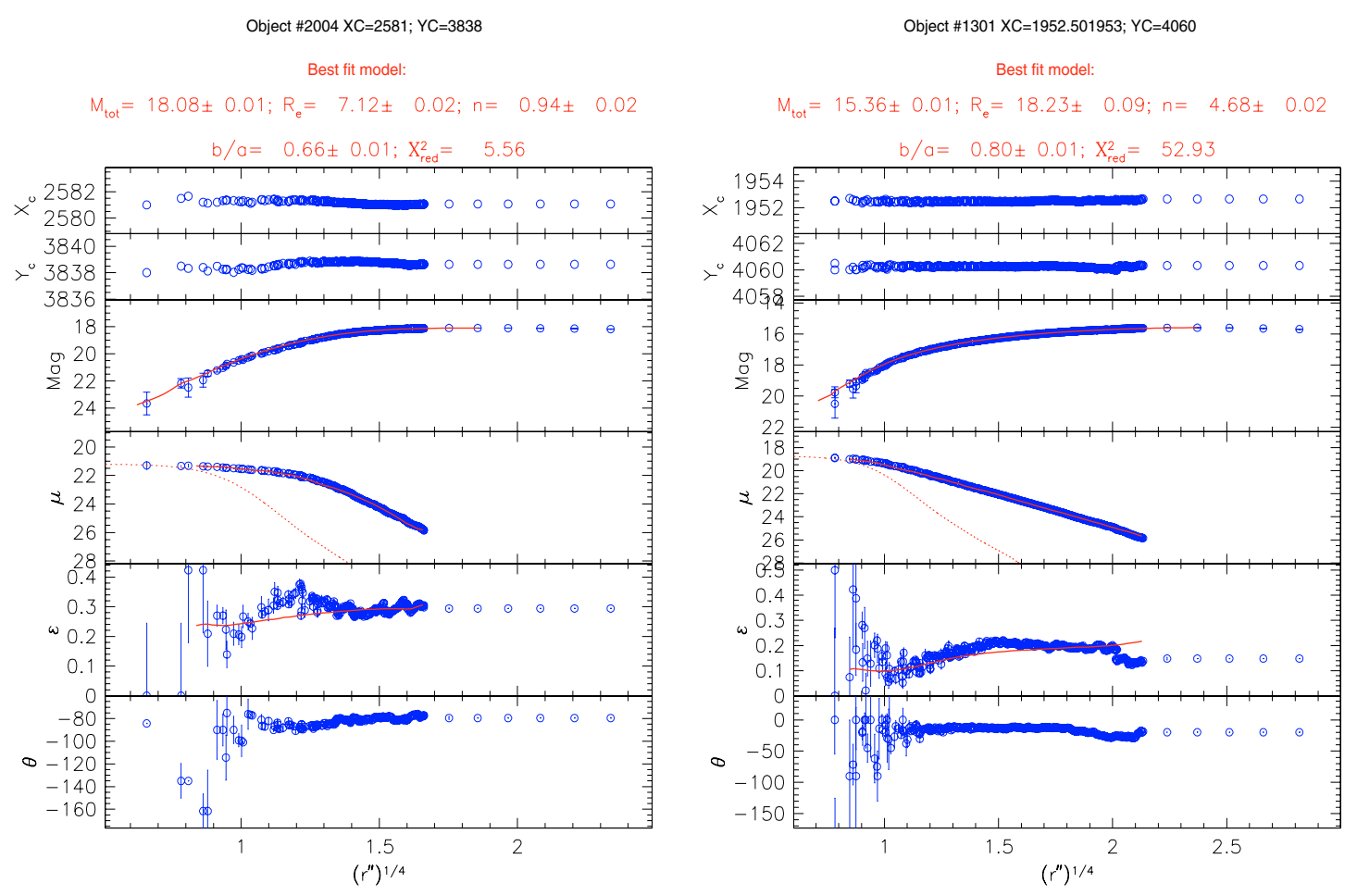

Fig. 3. Major- and minor-axis luminosity profiles obtained with GASPHOT, together with the corresponding best-fitting (convolved) Sersiclaw functions, for a spiral galaxy (left) and an elliptical galaxy (right) in the cluster A119. The very different Sersic indices correspond to the different morphological types. The solid lines represent the best-fit models found by GASPHOT, while the dotted lines indicate the PSF profiles.

For parameter $k$, we adopted the analytical approximation given by Ciotti (1991):

$k(n) \approx 2 n-\frac{1}{3}+\frac{4}{405 n}+\frac{46}{25515 n^{2}}+\frac{131}{1148175 n^{3}}$

which is valid in the range of physical interest for $n$ with a relative error of less than $10^{-6}$. In particular, we have $k=7.676$ for $n=4$ and $k=1.676$ for $n=1$ (the de Vaucouleurs and the exponential law, respectively).

The profile given in Eq. (1) is then convolved by the PSF previously obtained. The multi-Gaussian representation we have adopted for the PSF profile allows us to generalize the analytical approach provided by Trujillo et al. (2001). According to this formalism, the major- and minor-axis profiles of a $2 \mathrm{D}$, flattened (axial ratio $b / a$ ) Sersic function, convolved with a multi-Gaussian PSF are given by the following expressions:

$$
\begin{aligned}
& I_{\mathrm{maj}}(\xi)=I_{\mathrm{bkg}}+\sum A_{i} \frac{(1-\varepsilon)}{2 \pi \sigma_{i}^{2}} \int_{0}^{\infty} I\left(\xi^{\prime}\right) \xi^{\prime} \mathrm{d} \xi^{\prime} \\
& \times \int_{0}^{2 \pi} \exp \left(-\frac{\xi^{2}+\xi^{2}-2 \xi \xi^{\prime} \cos (\theta)+\left(\varepsilon^{2}-2 \varepsilon\right)\left(\xi^{\prime} \sin \theta\right)^{2}}{2 \sigma_{i}^{2}}\right) \mathrm{d} \theta \\
& I_{\min }(\xi)=I_{\mathrm{bkg}}+\sum A_{i} \frac{(1-\varepsilon)}{2 \pi \sigma_{i}^{2}} \int_{0}^{\infty} I\left(\xi^{\prime}\right) \xi^{\prime} \mathrm{d} \xi^{\prime} \\
& \times \int_{0}^{2 \pi} \exp \left(-\frac{\xi^{2}+\xi^{\prime 2}+2 \xi \xi^{\prime} \sin (\theta)+\left(\varepsilon^{2}-2 \varepsilon\right)\left(\xi^{\prime} \sin \theta-\xi\right)^{2}}{2 \sigma_{i}^{2}}\right) \mathrm{d} \theta
\end{aligned}
$$

where $\xi=R / R_{\mathrm{e}}, \varepsilon=1-b / a$, and $A_{i}$ and $\sigma_{i}$ are the amplitude and standard deviations of the Gaussians defining the PSF profile.
We then integrate Eqs. (3) and (4) along the major and minor axis respectively, and use the resulting growth curves to perform a simultaneous fit of the corresponding observed profiles with a Levenberg-Marquardt $\chi^{2}$ procedure.

For each galaxy, the best-fitting tool retrieves the total magnitude $m_{\mathrm{T}}$, the half-light radius $R_{\mathrm{e}}$, the Sersic index $n$, the axial ratio $b / a$, and the sky level $I_{\mathrm{bkg}}$, together with their formal $1 \sigma$ errors. Since the parameters of the model are strongly correlated each to the other, in order to derive correct estimates of derived quantities (such as the average surface brightness $\langle\mu\rangle_{\mathrm{e}}$ ), we also produce the full covariance matrix of the parameter set. Figure 3 shows typical examples of luminosity and structural profiles of galaxies, together with the corresponding best-fitting Sersic models.

\subsection{Tips and warnings}

GASPHOT constrains the search of the minimum $\chi^{2}$ within a parameter space limited both in the Sersic index $(0.5<n<8)$ and in the effective radius $\left(R_{\mathrm{e}}>0.7\right.$ pixels). This allows us to flag galaxies for which we know that the fit failed for some reason. Thus, besides the case when the covariance matrix of the parameters becomes singular, we consider that a failure of the best-fitting procedure occurs when at least one of the outputs coincides with some limiting value of the parameter space. Unfortunately, this procedure does not allow the user to identify the galaxies for which the fit found a local minimum of the $\chi^{2}$, giving unrealistic results. In the following we will call "failed fits" only the flagged objects - to be removed from the 
statistical analysis - while the local minima will still be present as outliers. We will see that the fraction of failed fits produced by GASPHOT is always very small.

While the outlined fitting procedure turns out to be quite stable, extraction of the underlying photometric profiles requires some care. In particular, since the isophotes of galaxies are obtained using SExtractor, our tool is obviously sensitive to the SExtractor configuration parameters. Special care must be applied when choosing the deblending parameters, as well as the detection and analysis threshold parameters (see the SExtractor manual for details). The former ones deeply influence the actual detection of blended objects and the geometry of their isophotes. Thus, it is strongly advised to look at the check-image provided by SExtractor before setting these parameters in the configuration file. The threshold parameters determine the surface brightness where the luminosity profiles of galaxies move from the isophotal to the Kron-like aperture regime (see Sect. 3.2). Since such a transition may produce some (usually small) discontinuity in the geometrical parameters (particularly in the axial ratio), the choice of the thresholds influences the shapes of the outer luminosity profiles, thus affecting the results of the fit. We have verified that a threshold of $2.5 \sigma_{\mathrm{bkg}}$ is enough to prevent the onset of noise-featured structures that artificially increase the ellipticity of the isophotes, thus securing a smooth transition from the isophotal to the aperture regime. We strongly suggest not using lower values of the thresholds, since this can bias the global parameters of the fit. Another delicate issue concerns the choice of mesh size for the background subtraction (see the SExtractor manual for details). Using the sky level $I_{\mathrm{bkg}}$ as a free model-parameter of the best-fitting procedure turns out to be an effective way to deal with those small residuals of the local background that are not properly removed by SExtractor, at least in the most common cases. However, this is potentially dangerous, particularly when dealing with blended objects, since any assumption about the sky level directly influences the shape of the outer luminosity profiles. Therefore we allow the user to limit the allowed range of values for the parameter $I_{\mathrm{bkg}}$. Such constraints could be used, for instance, when the user is confident that a good sky subtraction has already been performed on the image before GASPHOT running. Finally, we have verified that the effect of pixelization does not affect the fitting algorithm appreciably, at least down to an $F W H M$ of $\sim 1.5$ pixels. Therefore, for the sake of speed in running the program, we decided not to take this effect into account.

\subsection{Times}

Since our goal is to provide an automatic tool, effective on hundreds or even thousands of objects, computational speed is a must. On our machine (Dec-Alpha, 657 MHz-CPU/2 GybRAM) and with WF-INT $(6284 \times 6208)$ imaging, the time needed to obtain the photometric profiles and to derive the corresponding best-fitting model parameters ranges between 2 and 4 min per object - including the SExtractions - implying at least one day of CPU time to handle an image with about 600 objects. However, time is not linear to the number of galaxies. In fact, since the speed of profile extraction only depends on the image size, large images with a small number of galaxies are not efficiently handled by GASPHOT. For instance, analyzing a large image with a single object could still take several hours, because of the time needed to extract the profiles. In this sense, GASPHOT is optimized for use with a large dataset of galaxies to be analyzed automatically, and is not suited to dealing with single objects, which are better handled interactively or by using other tools (GALFIT).

\section{Testing on simulations}

In order to test the tool's performance, we started by simulating toy galaxies by means of the IRAF task mkobject. Such simulations have been used not only to test the numerical precision of both the convolution algorithm and the best-fitting procedure, but also to provide robust lower limits to the errors expected for real galaxies, which are usually far from having either pure Sersic-law luminosity profiles or constant axial ratios and position angles.

Different kinds of artificial frames have been produced for this first testing phase, each one optimized according to a different testing criterion: (i) single Sersic profile (de Vaucouleurs/exponential) galaxies positioned on a wide grid, in order to minimize possible blending effects; (ii) two-component (de Vaucouleurs + exponential) profile galaxies, again on a wide grid; (iii) crowded, cluster-like fields, including many blended objects. In any case the toy galaxies were generated in a wide range of size, magnitude, and axial ratio. The total number of toy galaxies on which we tested GASPHOT was about 15000.

Even though GASPHOT was conceived for general use, we produced our testing simulations to mimic the average observational conditions of the $V$-band imaging coming from the WINGS survey of galaxy clusters (Fasano et al. 2003), since this is the first application of GASPHOT we were interested in. Extension of the results obtained here to other kind of images will be discussed in Sect. 4.4.

Thus, in our mkobject simulations, we used the following typical WINGS values: (i) exposure time $=1200 \mathrm{~s}$; (ii) sky-level $=1 \mathrm{ADU} / \mathrm{s}$; (iii) pixel-size $=0.333$ arcsec; (iv) PSF $-F W H M=3$ pixels; (v) gain $=2.775 \mathrm{e}^{-} / \mathrm{ADU}$; (vi) ReadOut Noise $=6.5 \mathrm{e}^{-}$. Axial ratios $(b / a)$, apparent total magnitudes, and angular radii of the toy galaxies were randomly extracted so that they reproduced the observed flattening distributions of early- and late-type galaxies, as well as the average magnitude-radius relation obtained for the WINGS galaxies $\left(15<m_{\mathrm{T}}<22 ; 2<R_{\mathrm{e}}\right.$ (pixels) $<30$; average relation $\left.\log R_{\mathrm{e}}=3.06-0.12 m_{\mathrm{T}} ; \mathrm{rms}=0.15\right)$. Finally, to properly take the uncertainties arising from the convolution into account, 20 artificial stars were included in each simulation, and GASPHOT was used to model their Gaussian PSF profile (see Sect. 3.3).

\subsection{Simulations on grid}

For both de Vaucouleurs and exponential luminosity profiles, large $(6600 \times 6600)$ images were generated with mkobject, 
$n=4$

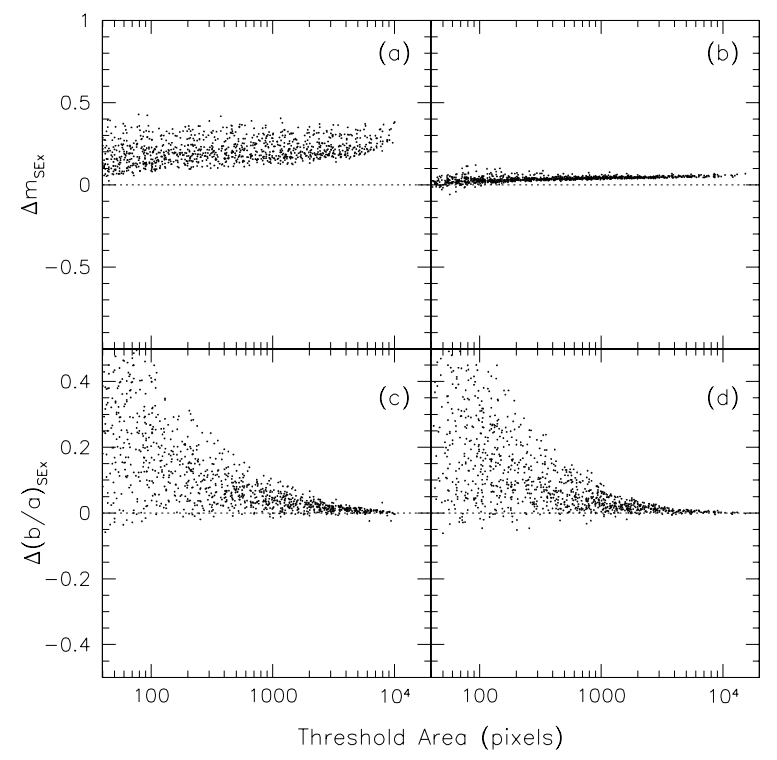

Fig. 4. Deviations of the total magnitudes (MAG_BEST) and axial ratios given by SExtractor from the input values of the simulations as a function of the threshold area. Because these galaxies are positioned on a grid and far from each other, the MAG_BEST is equivalent to the MAG_AUTO for all the objects.

each artificial image containing 1024 toy galaxies on a grid (mesh-size: 200 pixels) and 20 toy stars. Then we ran SExtractor to produce the reference catalogs.

Our simulations on the grid confirm the scatter and large bias that have been claimed by the authors themselves (Bertin \& Arnouts 1996) to affect the axial ratios and the total magnitudes provided by SExtractor, especially in the case of de Vaucouleurs luminosity profiles (Fig. 4; see also Franceschini et al. 1998).

Using the SExtractor catalogs as reference, we ran GASPHOT over the simulated images, first recording total magnitudes $\left(m_{\mathrm{T}}\right)$, half-light radii $\left(R_{\mathrm{e}}\right)$, Sersic indices $(n)$, and axial ratios $(b / a)$, and then comparing these values with the corresponding input (known) quantities. We checked the goodness of the retrieved parameters (that is, the difference from the original ones) versus the parameters themselves, considering acceptable values for the absolute differences $\Delta m_{\mathrm{T}}, \Delta R_{\mathrm{e}} / R_{\mathrm{e}}$, $\Delta n / n$, and $\Delta(b / a)$ those lower than $0.05,0.1,0.1$, and 0.05 , respectively. For toy galaxies with de Vaucouleurs profiles, we found that both the effective radii and the Sersic indices retrieved by GASPHOT were biased downwards $\left(\triangle R_{\mathrm{e}} / R_{\mathrm{e}}<-0.1\right.$ and $\Delta n / n<-0.1)$ when $R_{\mathrm{e}}<1.5$ pixels and $m_{\mathrm{T}}>19.5$, while no systematic over-running of the allowed tolerances were found for the average total magnitudes and axial ratios. Finally, all the average parameters retrieved by GASPHOT for exponential profile galaxies turned out to be well inside the tolerance bands. For both kinds of profiles, the bias and scatter obtained for recovered magnitudes and radii were always well below those obtained with SExtractor.

In Fig. 5 and hereafter, the differences between retrieved and input values of the parameters are shown as a function of the $2.5 \sigma_{\mathrm{bkg}}$ threshold area $\left(A_{\mathrm{thr}}\right)$ for both de Vaucouleurs
( $n=4$, left side) and exponential ( $n=1$, right side) profile galaxies. This choice has the advantage of making the analysis independent of most observational parameters (exposure time, zero point, noise), the only left dependence being that on the $F W H M$ of the PSF in pixels (see Sect. 4.4). The filled strips in Fig. 5 include $50 \%$ of the objects and are obtained connecting the quartiles (positive and negative) around the local median difference, binned over the threshold area. The full lines connect the median error bars provided by GASPHOT in the different bins. They are clearly consistent with the real scatter of the points. The biases of $R_{\mathrm{e}}$ and $n$ that we found for small and faint galaxies in the case of $n=4$ luminosity profiles translate onto the low threshold area objects in the left side plots of Fig. 5.

For each bin of $A_{\mathrm{thr}}$, the histograms in Fig. 6 report the fraction of objects for which the differences between retrieved and input values of all parameters are within the tolerance bands for both de Vaucouleurs (solid line) and exponential (dashed line) profile galaxies. Obviously, these fractions increase at increasing $A_{\text {thr }}$, the exponential profiles reaching saturation much more rapidly than the de Vaucouleurs profiles. Retrieving the original parameters for high Sersic indices is indeed expected to be hard, because of the presence of more extended wings in the surface brightness profiles, which makes the extrapolation more sensitive to errors. From Fig. 6 it turns out that a lower limit of $\sim 250$ pixels for $A_{\text {thr }}$ is required by GASPHOT in order to provide reliable estimates of the parameters. Below this limit, for more than $50 \%$ of the de Vaucouleurs profile galaxies in the smallest area bin, at least one of the parameters is retrieved outside the allowed tolerance band. Although obtained for WINGS-like imaging, this result should be regarded as a general feature of GASPHOT, independent of any observational parameter, apart from the $F W H M$ in pixels. We briefly discuss this point in Sect. 4.4.

In Cols. 1 and 3 of Table 1 (first and second rows of each parameter) the median deviations of the model parameters retrieved by GASPHOT for grid toy galaxies with $A_{\mathrm{thr}}>$ 250 pixels are reported for exponential and de Vaucouleurs profiles, together with the corresponding semi-interquartile ranges $( \pm)$.

\subsubsection{Failed fits and outliers}

For $A_{\mathrm{thr}}>250$ pixels, the fraction of failed fits (see Sect. 3.5) in our simulations on the grid turns out to be almost negligible $(<0.2 \%)$. In the following we remove the failed fits from the statistical analysis.

We considered outliers with respect to a given parameter those object with a deviation from the input value of the parameter larger than two times the allowed tolerance. In Cols. 1 and 3 of Table 1 (third and fourth rows of each parameter), we report both the percentage of outliers with threshold area greater than 250 pixels and their median absolute distance from the input value of the parameter. Table 1 shows that, for our simulations on grid, the fraction of outliers with threshold area greater than 250 pixels, as produced by GASPHOT ranges between $0.1 \%$ and $5 \%$ (larger fractions for $n=4$ ), while the median deviation of outliers ranges between 1.3 and 3.8 times the 


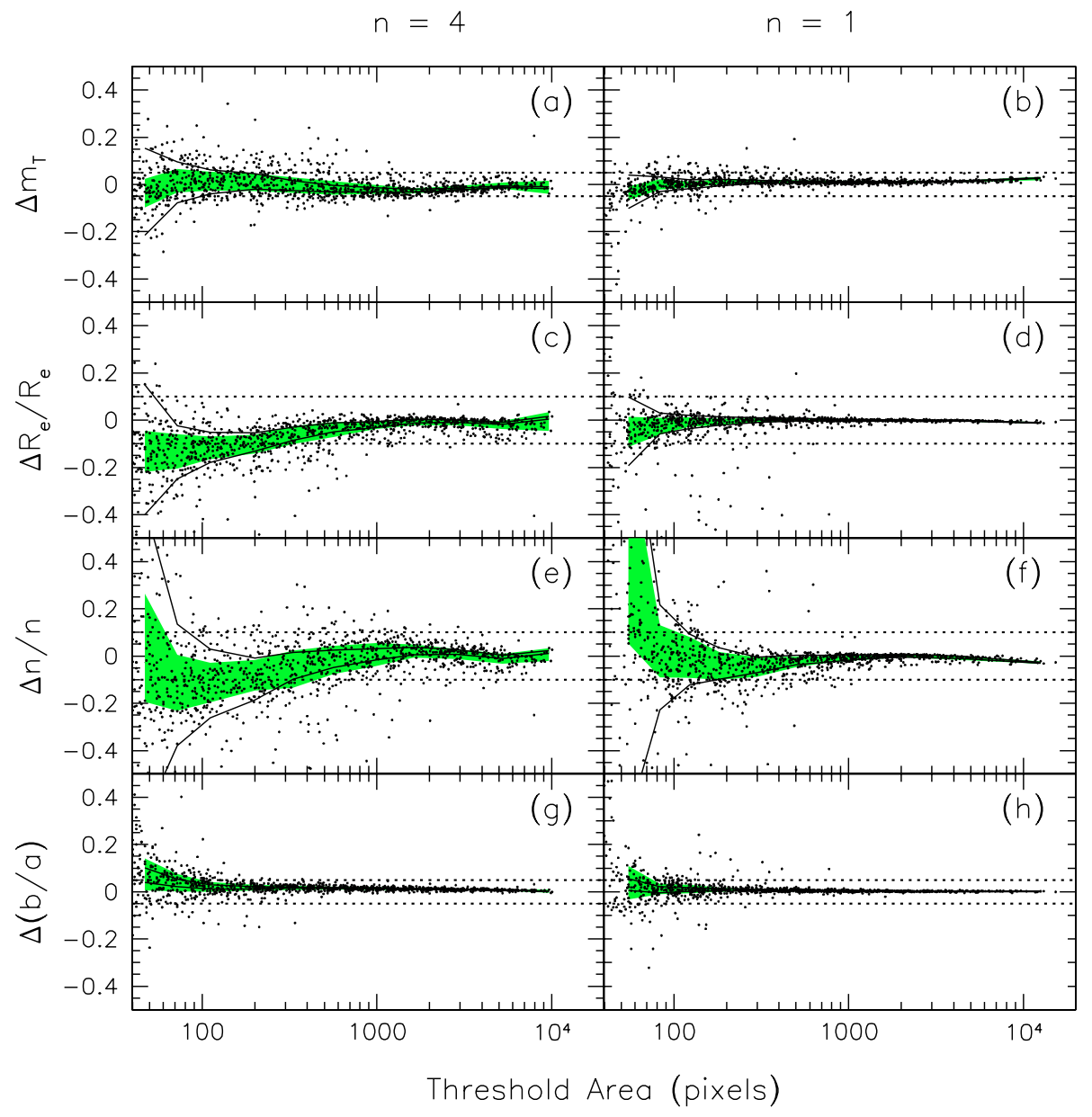

Fig. 5. Differences between retrieved and input values of the parameters for both de Vaucouleurs $(n=4$, left side) and exponential $(n=1$, right side) profile galaxies, as a function of the $2.5 \sigma_{\mathrm{bkg}}$ threshold area. The filled strips include $50 \%$ of objects and are obtained by connecting the quartiles (positive and negative) around the local median difference, binned over the threshold area. The full lines connect the median error bars provided by GASPHOT in the different bins.

Table 1. Photometric parameters retrieved by GASPHOT, GALFIT, and GIM2D for all the simulations and clusters presented in this paper. For all quantities we give the median deviations of the model parameters, as well as the corresponding semi-interquartile ranges. All quantities are evaluated using only galaxies with $A_{\mathrm{thr}}>250$ pixels.

\begin{tabular}{|c|c|c|c|c|c|c|c|c|c|}
\hline & \multicolumn{4}{|c|}{ Grid } & \multicolumn{3}{|c|}{ Blended } & \multicolumn{2}{|c|}{ Real } \\
\hline & \multicolumn{2}{|c|}{$n=1$} & \multicolumn{2}{|c|}{$n=4$} & \multirow[b]{2}{*}{$\begin{array}{l}\text { Gasphot } \\
\text { (5) }\end{array}$} & \multirow[b]{2}{*}{$\begin{array}{c}\text { Galfit } \\
(6)\end{array}$} & \multirow[b]{2}{*}{$\begin{array}{c}\operatorname{Gim} 2 d \\
(7)\end{array}$} & \multirow[b]{2}{*}{$\begin{array}{c}\text { Gasphot } \\
(8)\end{array}$} & \multirow[b]{2}{*}{$\begin{array}{c}\text { Galfit } \\
(9)\end{array}$} \\
\hline & $\begin{array}{c}\text { Gasphot } \\
\text { (1) }\end{array}$ & $\begin{array}{l}\text { Galfit } \\
(2)\end{array}$ & $\begin{array}{c}\text { Gasphot } \\
\text { (3) }\end{array}$ & $\begin{array}{l}\text { Galfit } \\
(4)\end{array}$ & & & & & \\
\hline$\Delta m_{\mathrm{T}}$ & 0.010 & -0.011 & -0.019 & -0.023 & 0.003 & -0.017 & 0.109 & 0 & -0.047 \\
\hline$( \pm)$ & $(0.005)$ & (0.003) & (0.018) & (0.009) & $(0.021)$ & (0.023) & $(0.035)$ & $(0.067)$ & $(0.154)$ \\
\hline$\% m_{\mathrm{T}}^{\text {out }}$ & 0.3 & 0 & 3 & 0 & 4.8 & 8.5 & 59.1 & 14 & 10 \\
\hline$\Delta m_{\mathrm{T}}^{\text {out }}$ & 0.19 & 0 & 0.13 & 0 & 0.14 & 0.24 & 0.15 & 0.44 & 1.27 \\
\hline$\overline{\Delta R_{\mathrm{e}} / R_{\mathrm{e}}}$ & -0.004 & 0.004 & -0.030 & 0.012 & 0.002 & 0.010 & 0.007 & -0.057 & -0.143 \\
\hline$( \pm)$ & $(0.003)$ & $(0.005)$ & (0.024) & (0.019) & (0.009) & $(0.040)$ & $(0.036)$ & $(0.101)$ & $(0.24)$ \\
\hline$\% R_{\mathrm{e}}^{\text {out }}$ & 0.8 & 0 & 1.5 & 0 & 3.5 & 5.3 & 5.4 & 12 & 7 \\
\hline$\Delta R_{\mathrm{e}} / R_{\mathrm{e}}^{\text {out }}$ & 0.35 & 0 & 0.13 & 0 & 0.35 & 0.46 & 0.54 & 0.46 & 1.03 \\
\hline$\Delta n / n$ & -0.008 & 0.030 & 0.002 & 0.067 & 0.002 & 0.045 & 0.07 & - & - \\
\hline$( \pm)$ & $(0.013)$ & $(0.015)$ & (0.037) & (0.029) & (0.019) & $(0.052)$ & $(0.06)$ & - & - \\
\hline$\% n^{\text {out }}$ & 2.3 & 0 & 5 & 1.4 & 5.3 & 7.7 & 18.2 & - & - \\
\hline$\Delta n / n^{\text {out }}$ & 0.35 & 0 & 0.29 & 0.23 & 0.35 & 0.27 & 0.43 & - & - \\
\hline$\Delta b / a$ & 0.003 & 0.000 & 0.011 & -0.002 & 0.006 & -0.001 & -0.05 & - & - \\
\hline$( \pm)$ & $(0.002)$ & (0.006) & (0.005) & (0.004) & (0.006) & (0.003) & $(0.07)$ & - & - \\
\hline$\% b / a^{\text {out }}$ & 0.2 & 0 & 0.1 & 0 & 1.0 & 1.6 & 12.8 & - & - \\
\hline$\Delta b / a^{\text {out }}$ & 0.12 & 0 & 0.12 & 0 & 0.16 & 0.23 & 0.13 & - & - \\
\hline
\end{tabular}




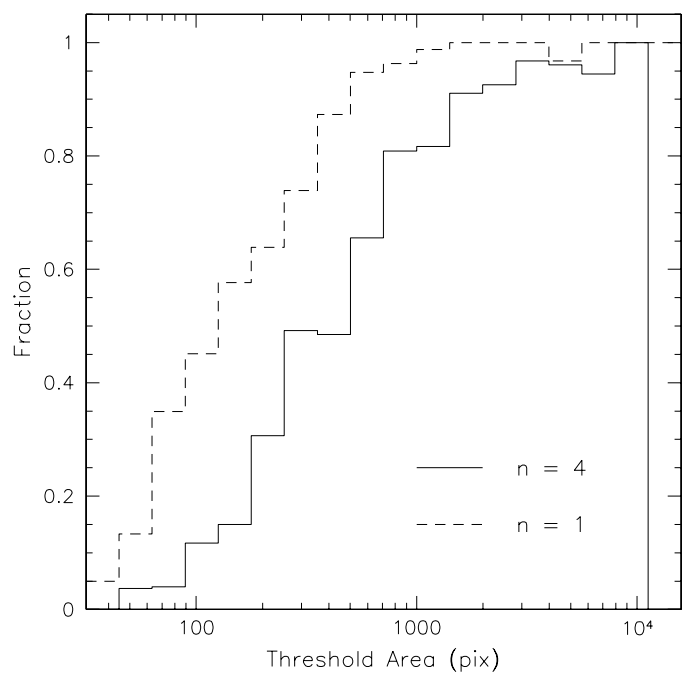

Fig. 6. Fraction of objects for which the differences between retrieved and input values of all parameters lie within the tolerance bands for both de Vaucouleurs (solid line) and exponential (dashed line) profile galaxies, as a function of the threshold area.

allowed tolerances (larger values for $n=1$ ). Some of these outliers are very likely due to a residual amount of blending in our test images; we put 1024 galaxies in a $6600 \times 6600$ image, so it may happen that some large objects partially overlap with the neighbours. Some other cases are very likely due to the numerical approximations involved in the integration, as well as to the pixelization effect, which may introduce unpredictable instabilities and/or biases in the $\chi^{2}$. Most of them are very flattened objects $(b / a \sim 0.1)$ for which the axial ratio is not well retrieved. We will see in the next sections that both the fraction of outliers and their average deviation increase when dealing with simulated, blended objects and with real galaxies.

\subsection{Composite profiles}

The luminosity profiles of real galaxies are well known for not following simple Sersic's laws. Deviations from this kind of profile may involve isophotal twisting, variation of ellipticity with radius, bar and lens components, double nuclei, asymmetric features, and so on. The simplest situation that can be reproduced by means of simulations, and by far the most common in real galaxies, is that of a multi-component galaxy with a de Vaucouleurs bulge plus an exponential disk. We wanted to test how well GASPHOT can retrieve the interesting photometric parameters (that is, magnitudes and optical radii) of these kind of galaxies, by fitting their luminosity profiles with simple Sersic's laws, which are not representative of the whole profile. We also wanted to see if the Sersic index $n$ brings any correlation with the bulge/disk ratio.

In order to achieve these results, we produced a simulation similar to those described in the previous section, with 1000 galaxies positioned on a grid, thus minimizing the blending. Each object was built as the superposition of a de Vaucouleurs and an exponential profile with different axial ratios, magnitudes, and optical radii, but with the same position angle. In the following we refer to the luminosity ratio

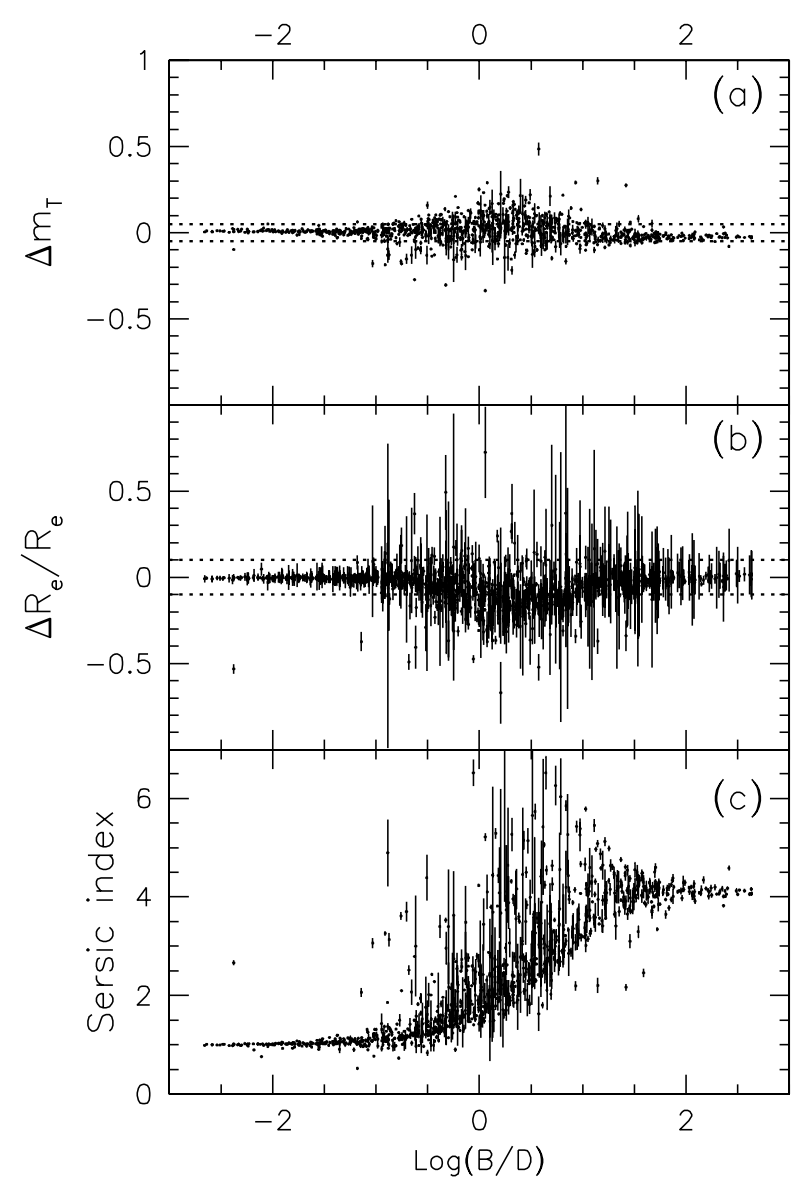

Fig. 7. Deviations of magnitudes (panel a)) and radii (panel b)) retrieved by GASPHOT from the input values of the simulation of twocomponent (de Vaucouleurs+exponential) galaxies, as a function of the $B / D$ ratio. As expected, the deviations of the retrieved parameters from the real ones are strongly correlated with the deviation of the profile from a pure Sersic law. Panel c) shows the correlation between the Sersic index $n$ found by GASPHOT and the $B / D$ ratio.

of the de Vaucouleurs to the exponential component as the "bulge-to-disk ratio", thus following the naive interpretation of the two components, even if as a rule other physical possibilities may be described by the same combination of components.

We did not put any more constraints on the parameters, which were allowed to vary randomly in the space of interest. This means that some of the toy galaxies have properties that are quite uncommon in the real galaxies, like a bulge with a larger radius than the disk radius, or an apparent flattening that is higher for the de Vaucoulers component than for the exponential one. This allows us to estimate the deviations of the retrieved parameters from the original ones in almost any possible situation, thus providing an upper limit on the errors given by the procedure.

As expected, the deviations of the retrieved parameters from the real ones strongly correlate with the deviation of the profile from a pure Sersic law. For this reason, in panels (a) and (b) of Fig. 7 we show these deviations versus the "bulgeto-disk ratio" of the toy galaxies.

The worst case is obviously when the bulge and disk components have similar luminosities, at which point both the 
luminosity and the radius tend to be underestimated. When we limited ourselves to the range $0.1<B / D<10$, we found $\Delta m_{\mathrm{T}} \approx 0.022$ (semi-interquartile $=0.04$ ) and $\Delta R_{\mathrm{e}} / R_{\mathrm{e}} \approx-0.06$ (semi-interquartile $=0.06)$, irrespective of the threshold area. This means that we can put robust upper limits on $\Delta m(\approx 0.1)$ and $\Delta R_{\mathrm{e}} / R_{\mathrm{e}}(\approx 0.15)$, even in the case of non-Sersic profiles.

In the bottom panel of Fig. 7, we show the correlation between the Sersic index $n$ and the $B / D$ ratio. It is clear that a correlation does exist, even if there is still a large scatter in the intermediate range $0.01<B / D<10$. Such scatter is expected, because the best-fit Sersic index depends not only on the magnitude ratio, but also on the scale length ratio of the two components.

In conclusion, we found that, within the mentioned errors, the photometric parameters $m_{\mathrm{T}}$ and $R_{\mathrm{e}}$ appeared to be robust enough for statistical applications, even in the presence of strongly non-Sersic profiles. On the other hand, the Sersic index turned out to be loosely correlated with the $B / D$ ratio and such a correlation could still be useful in a statistical sense.

\subsection{Blended galaxies}

Apart from the deviations from a pure Sersic profile, which we analyzed in the previous section, the most serious problem in deriving the photometric parameters automatically arises from the isophotal blending among projected and/or physical companion galaxies, as well as among interacting/merging systems. All automatic tools are likely to have problems with these kinds of objects, which are expected to be very common in dense systems like clusters or groups. The most disturbing characteristic of the blended objects is that an automatic tool might provide unreliable results at least for a fraction of them, i.e. wrong parameters with small error bars.

The best way to handle these objects would obviously be to create an appropriate mask for each object in order to remove the companions from the image. Unfortunately, this procedure needs an amount of interactivity unsuitable to this kind of work, so it can hardly be implemented automatically. We decided to rely on the deblending performed by SExtractor during the construction of the isophotes, which has the obvious advantage of already being completely automatic and very fast. In doing that we must, however, keep in mind the limitations of such a procedure. Actually, SExtractor performs the deblending by assigning each pixel inside a blended region to just one of the blending objects. While this procedure turns out to be robust enough as far as the aperture magnitudes of the isophotes are concerned, it does not preserve their geometrical parameters, that is, area, radius, axial ratio, and position angle. As a consequence, the photometric profiles to be fitted are artificially distorted. In addition, the luminosity profiles of blended galaxies are contaminated by a non-constant background due to the contribution of the companion galaxies.

We had no simple way of correcting for the distortions of the profiles induced by the blending. Therefore, we simply decided to discard from the fit those isophotes that have been flagged by SExtractor as "blended" (that is, have a FLAG > 2, see the SExtractor manual for details). This procedure has

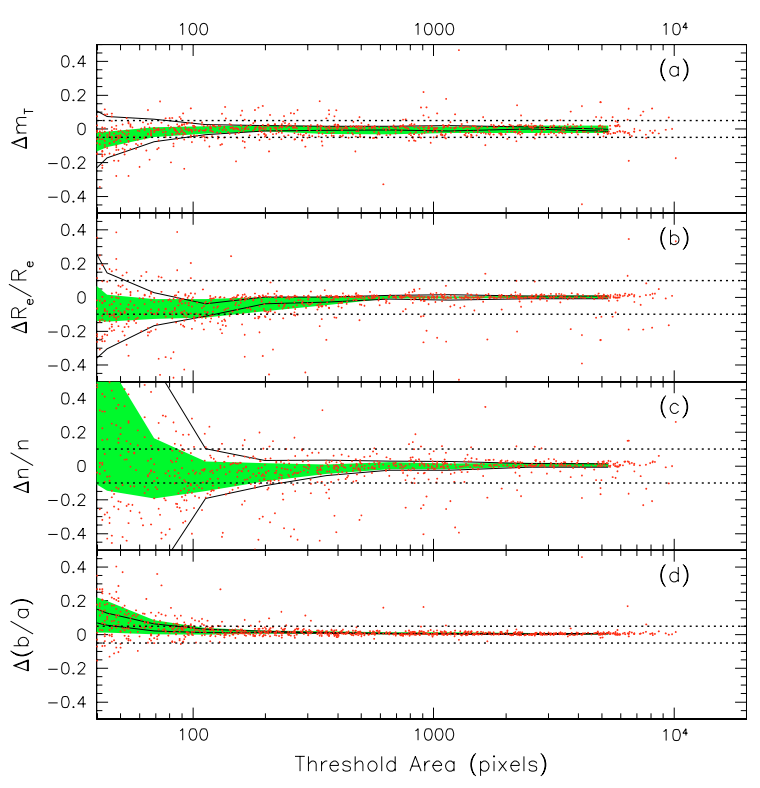

Fig. 8. Same as Fig. 5, but for a simulation that includes heavily blended objects.

the drawback of introducing large error bars for the heavily blended objects for which only a small fraction of the luminosity profile may be available, but it also has the crucial advantage of avoiding deceptive results.

In order to analyze the effect of blending on the performances of GASPHOT, we produced an artificial cluster composed of galaxies with pure de Vaucouleurs and exponential profiles. At variance with the previous tests, we simulated a King density profile for the cluster rather than putting the toy galaxies on a grid. The other observational parameters were the same as in the previous sections. The results are reported in Col. 5 of Table 1 and illustrated in Fig. 8, where de Vaucouleurs and exponential profile galaxies are shown together. For most objects the differences between the input parameters and the corresponding ones provided by GASPHOT still follow the relations found in the previous simulations, with the same biases and scatters depending on the luminosity profile population (de Vaucouleurs/exponential). In addition, we note that: (i) there is a significant population of heavily blended objects for which only a small fraction of the photometric profile was used in the fitting procedure. This produced large uncertainties in the output parameters, which still make them consistent - that is, within the output error bars - with the corresponding input parameters; (ii) there is a small fraction of galaxies (about 1\%) for which the blending is so heavy that even the axial ratio is not well recovered. For these objects the retrieved photometric parameters are usually not consistent with the input ones. From Table 1 it is also clear that both the fraction of outliers and their average deviation from the input parameters are increasing with respect to the simulations on the grid.

In conclusion, the results are quite robust in a statistical sense. For more than $95 \%$ of the objects, the precision of the recovered parameters is within the limits given in previous sections for isolated objects. For most of the blended objects, 
the blending produces large error bars instead of non-realistic parameters.

\subsection{Generalizing the results of simulations}

The simulations illustrated in the previous sections have shown that, within wide ranges of size and magnitude, the agreement between the input and the recovered parameters mainly depends on the threshold area, defined as the number of pixels having counts greater than 2.5 times the rms of the background. This implies that, given the pixel size and the FWHM of the PSF, the other observational parameters (like photometric zero point, exposure time, gain, etc.) influence the performances of GASPHOT only insofar as they change the threshold area of galaxies. Thus, besides the spatial resolution (redshift), the only left observational parameter that can affect the performances of GASPHOT is the FWHM of the Point Spread Function in pixels. In order to explore how the results of GASPHOT depend on this parameter, we repeated the above simulations using two different PSFs, with FWHM half (1.5 pix) and twice (6 pix) the value adopted in the previous tests. We conclude that the performances of GASPHOT as a function of $A_{\text {thr }}$ are qualitatively unchanged. As a rule of thumb, performances similar to those illustrated in the previous plots are found by increasing the threshold area by $\approx \pi \Delta\left(r_{\mathrm{PSF}}^{2}\right)$, where $r_{\mathrm{PSF}}$ is the $F W H M$ radius of the PSF.

GASPHOT always assumes that the PSF is a symmetric, perfectly circular multi-Gaussian. Unfortunately, this is rarely the case. In order to check the performance of the tool with a more complicated PSF, we rebuilt the "grid" simulation using a PSF with an axial ratio of 0.8 and a position angle of $45^{\circ}$. The results were qualitatively very similar to those shown in Sect. 4.1. In particular, bias and scatter of all parameters turned out to increase by less than $50 \%$. For this reason, we estimated that the robustness of the tool is not hampered by moderately non-circular PSF. A somewhat higher effect was found for very small objects $\left(A_{\text {thr }}<150\right)$, but in this case GASPHOT is not supposed to be able to provide reliable values for the parameters (see last paragraph of Sect. 4.1 and Fig. 6).

An additional point to consider is the generalization of the results from the simulations with respect to the Sersic index. Although the simulations have actually been performed using Sersic indices $n=1$ and 4 , there is no reason to believe that the performances of GASPHOT inside this interval could be worse than those shown in Sect. 4. Instead, looking at the behavior of these performances when one moves from $n=1$ to $n=$ 4 , we might guess that, for $n>4$, they could progressively deteriorate.

\section{Testing on real galaxies}

Testing on simulations has the advantage that the true parameters of galaxies are known a priori. On the other hand, even analyzing a wide range of situations (as we tried to do) may lead to strong underestimation of the true errors involved, simply because toy galaxies are regular by definition, while we know that real galaxies may have asymmetries, irregularities in the profiles, features, etc. Therefore, it is mandatory that
GASPHOT undergoes a final, more severe test: real galaxies. Since in this case the true parameters are obviously unknown, we must rely on previous studies in which the photometric parameters were evaluated interactively, working on every single object. This will of course bring additional scatter into the results, since even the parameters we are checking against are certainly affected by both random and systematic (often unknown) errors.

We concentrated on a set of four Abell clusters (A1069, A2111, A2670, A3125) for which photometric parameters of 231 early-type galaxies have been already published (Fasano et al. 2002). We ran GASPHOT on the same $R$-band images which were used by the authors of the previous papers for their photometry and compared the results in Col. 8 of Table 1 and Fig. 9. Since this dataset only includes early-type galaxies, we also ran GASPHOT on the HST imaging of an additional cluster (Abell 370) that was part of the MORPH project (Smail et al. 1997). For this cluster, magnitudes of most galaxies were derived using SExtractor, while detailed surface photometry was available only for nine objects (Ziegler et al. 1999), which we used in the comparison with GASPHOT. On the other hand, since the galaxy sample includes in this case many late-type objects, we considered the MORPHS classifications of the whole Abell 370 galaxy sample in investigating the correlation between Sersic index and morphological type shown in Fig. 10. The comparison with data in Fasano et al. (2002) and Smail et al. (1997) has been restricted to magnitudes and radii, since the Sersic indices and the axial ratios given in those papers are obtained using procedures that were too different from those used by GASPHOT.

As expected, even though the average differences between automatic (GASPHOT) and interactive parameters are small, the scatter turns out to be much larger than in the case of simulations (see Table 1 and leftmost panels in Fig. 9). Moreover, in this case the error bars associated to the retrieved parameters are less meaningful, since they actually account for the random errors only, and do not consider the uncertainties arising from peculiar features and irregularities of the real galaxies. Indeed, part of the scatter is due to the fact that the reference magnitudes and radii in Fasano et al. (2002) are obtained using just de Vaucouleurs and exponential extrapolations of the luminosity profiles of galaxies, while the Sersic index in GASPHOT is a free parameter. We tried to remove this systematic effect by linearly correlating the retrieved values of the Sersic index with magnitude and radius differences (see central panels in Fig. 9). The rightmost panels in the figure show the residual differences as a function of the threshold area. It is clear that, in spite of the statistical correction, the scatter remains large and increases when increasing the threshold area of galaxies. In particular, both the total luminosity and the effective radius of very large galaxies seem to be systematically underestimated by GASPHOT in comparison with the values provided by interactive surface photometry. Visual inspection and individual checks of large area galaxies led us to conclude that these effects reflect the increasing difficulty (by both GASPHOT and interactive tools) of describing and extrapolating the luminosity profiles of larger and larger galaxies in clusters, due to blending with smaller companions. Some of these objects are 


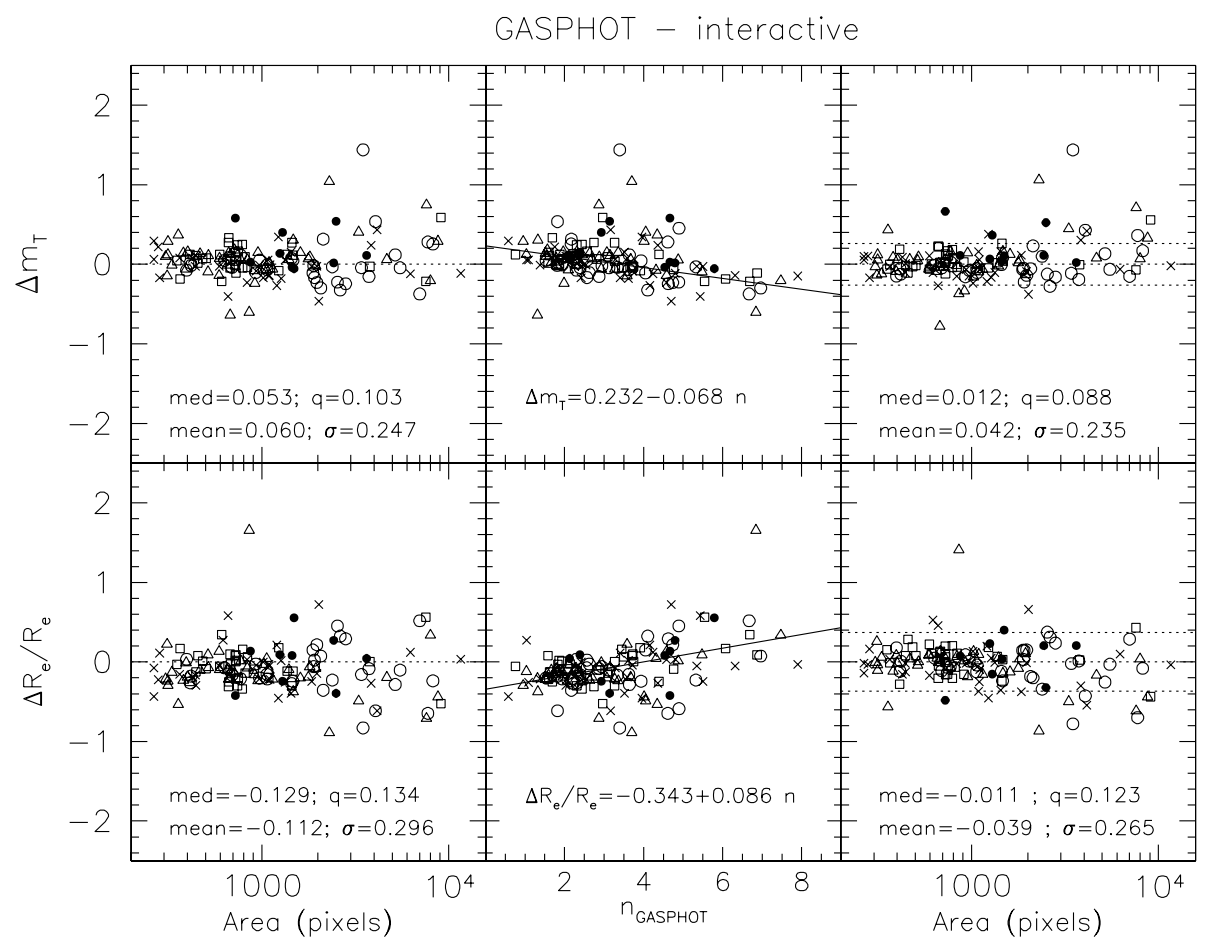

Fig. 9. Left panels: differences of magnitude $\left(\Delta m_{\mathrm{T}} ;\right.$ upper panel $)$ and effective radius $\left(\Delta R_{\mathrm{e}} / R_{\mathrm{e}} ;\right.$ lower panel) between GASPHOT and interactive surface photometry of real galaxies as a function of the threshold area. Crosses, open squares, circles, and triangles refer to the clusters A1069, A2111, A2670, and A3125 (from Fasano et al. 2002), respectively, while filled circles refer to the MORPHS cluster A370. Middle panels: systematic trends of $\Delta m_{\mathrm{T}}$ and $\Delta R_{\mathrm{e}} / R_{\mathrm{e}}$ as a function of the Sersic index by GASPHOT (see text for an explanation of this effect). Right panels: residual differences $\Delta m_{\mathrm{T}}$ and $\Delta R_{\mathrm{e}} / R_{\mathrm{e}}$ after statistical correction for the relations shown in the middle panels. The dotted lines in the plots correspond to differences of three times the semi-interquartile ranges.

cD galaxies or central members of subgroups. These objects look like elliptical galaxies (with the de Vaucouleurs profile) embedded in larger and flatter halos. Because of the presence of many nearby smaller companions, the outer isophotes of these objects are often flagged as "blended" by SExtractor, so that GASPHOT may sometimes just fit the de Vaucouleurs part of the profiles, thus giving smaller luminosities and optical radii than the ones given in Fasano et al. (2002). This problem actually concerns a few galaxies per cluster and involves the very definition of $\mathrm{cD}$ galaxies including the decision about where these objects end. From a statistical point of view, we are confident that the GASPHOT parameters of these objects can still be considered as meaningful. It is reassuring that by using such parameters, these galaxies still lie on the Kormendy relation $\mu_{\mathrm{e}}-R_{\mathrm{e}}$.

Besides these systematic effects, a small number of genuine outliers are present in the rightmost plots of Fig. 9. Most of them are found either to have heavily structured, non-Sersic profiles (i.e. rings, bars, etc.) or to be part of strongly blended systems. For this kind of comparison, in which the quantities to be compared are obtained using quite different procedures, we realistically consider those objects as outliers for which the absolute difference is greater than three times the semi-interquartile range (dotted lines in the rightmost panels of Fig. 9). From Col. 7 of Table 1 (third and fourth rows) it is clear that both the fraction of outliers and their average deviation from the input parameters are much larger than those found for simulations.

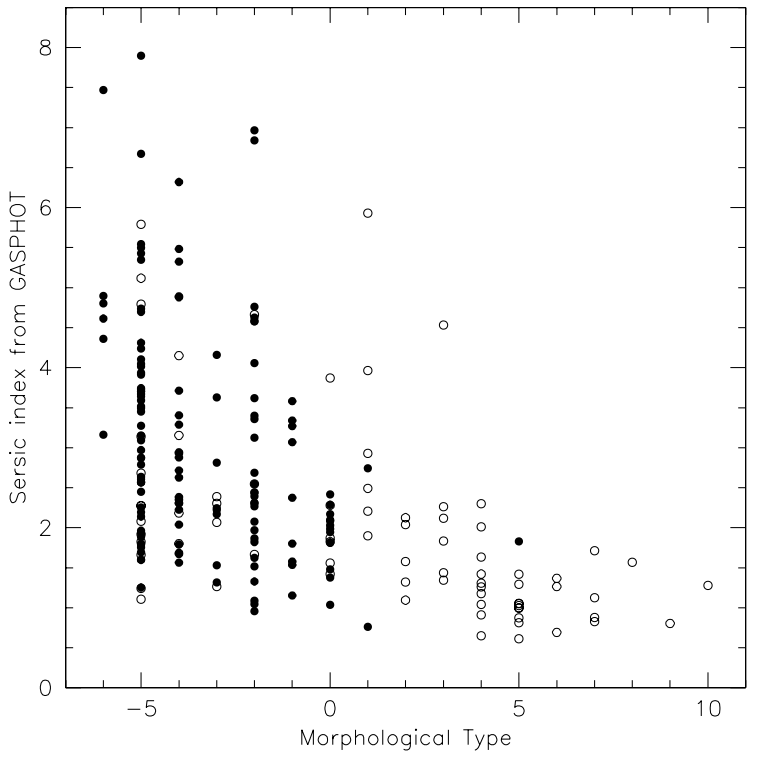

Fig. 10. Correlation between the Sersic index $n$ found by GASPHOT and the morphological type $T$ as given by Fasano et al. (2002) by visual inspection.

Figure 10 illustrates the correlation between the Sersic index $n$ provided by GASPHOT and the de Vaucouleurs morphological type $T$ given in Fasano et al. (2002) and Smail et al. (1997). Clearly, there is no univocal correspondence between morphology and Sersic index $n$. This is basically a 
measure of the concentration of the light profile and, in fact, for purely Sersic profiles, there is an analytical relation between the Sersic and the concentration indices (Trujillo et al. 2001). On the other hand, it is well known that the concentration index alone is not enough for a morphological classification and that at least a second parameter (asymmetry, color, etc.) is required (Abraham et al. 1994). Figure 10 shows that early-type galaxies actually share a wide range of values for $n$. Such large variance is expected, since D'Onofrio et al. (1994) have shown that the Sersic index of early-type galaxies increases when increasing the effective radius. We stress that for this reason the Sersic index alone cannot be used for morphological classification. In a forthcoming paper (Fasano et al. 2005), the Sersic index $n$ will be used, in combination with a number of additional ad hoc indicators, to give robust automatic estimates of the morphological classification of galaxies.

\section{Comparison with GALFIT and GIM2D}

To estimate the relative goodness of the results provided by GASPHOT, we compared them with those produced by GALFIT (Peng et al. 2002) for the same set of both artificial and real galaxies. We also compared with GIM2D (Marleau \& Simard 1998), but in this case we limited the comparison to the cluster-like simulation (blended galaxies).

At variance with GASPHOT, both GALFIT and GIM2D perform a $2 \mathrm{D}$ fitting of the galaxy images, trying to reproduce the observed light distribution by combining different models, including the Sersic law. To compare these two tools with GASPHOT in a consistent way, we forced them to use a single Sersic law in all cases.

Also at variance with GASPHOT, GALFIT processes galaxies one at a time, using a configuration file that contains some image parameters and some guesses for the multicomponent model parameters: magnitude, scale radius, axis ratio, etc. of each component. At the end, the tool gives a model image, a residual image, and a text file containing the best-fit parameters. Thus, to perform the comparison between GALFIT and GASPHOT for a large number of galaxies we built an IDL driving routine, using SExtractor to determine the initial guess of the parameter set. This routine prepares the configuration file of each galaxy, runs GALFIT, and writes results on the output file.

In the case of GIM2D, we used the tools gscripter and xgal to produce a small postage of each galaxy in the simulated image and the gimfit2d tool to fit the corresponding galaxy light distribution with a simple Sersic model. Galaxy images are symmetrized by GIM2D before fitting.

We ran GALFIT on the same simulations used for GASPHOT, i.e. de Vaucouleurs and exponential profile galaxies on a grid, and a galaxy cluster with a King density profile. In addition, we ran GALFIT on the real galaxies in the clusters used to test GASPHOT (Sect. 5). The results for simulations on a grid, for artificial cluster (blending), and for real galaxies are summarized in Cols. 2, 4, 6, 9 of Table 1 and illustrated in Figs. 11-13, respectively.

For the galaxies simulated on a grid, at variance with GASPHOT, the percentage of outliers produced by GALFIT turns out to be negligible, while the results obtained by GALFIT are qualitatively similar to those retrieved by GASPHOT, as far as bias and scatter are concerned, apart from a much larger bias in the Sersic index determined by GALFIT (see Cols. 2 and 4 of Table 1 and Fig. 11). Instead, when we move from pure Sersic profile galaxies to more complex systems, such as blended objects or real galaxies, GASPHOT appears to be noticeably more precise and more robust than GALFIT (see Cols. 6 and 9 of Table 1 and Figs. 12 and 13).

In particular, the performances of GASPHOT in recovering effective radii and Sersic indices of blended objects and real galaxies turn out to be clearly better than those of GALFIT in terms of the bias, scatter, and fraction of outliers. We interpret this result as a consequence of the different approaches adopted. On one hand, since we based our pipeline on SExtractor, we have the advantage of relying on the SExtractor deblending procedure, making GASPHOT more precise when dealing with interacting objects. On the other hand, the 1D approach of GASPHOT makes our results more robust and less sensitive to the many peculiarities of the real objects. This result was somehow expected, since GALFIT is essentially conceived for detailed fitting of individual objects, rather than for blind surface photometry of hundreds (or even thousands) of objects. An additional indication supporting this conclusion is given in Fig. 14, where magnitudes and radii obtained by GASPHOT and GALFIT for each object of the sample of real galaxies are compared with the corresponding median values among GASPHOT, GALFIT, and interactive photometry. Obviously, the scatter of each given tool (GASPHOT and GALFIT) in these plots is roughly proportional to the fraction of galaxies for which the $m_{\mathrm{T}}$ and $R_{\mathrm{e}}$ determinations of the tool itself are different from the remaining two determinations. Note that we removed the zeros of $\Delta m_{\mathrm{T}}$ and $\Delta R_{\mathrm{e}} / R_{\mathrm{e}}$ both from Fig. 14 and from the statistics.

Although a complete comparison with GIM2D is beyond the scope of this paper, we also checked the performances of this package on our simulated galaxies. However, since GIM2D turned out to be remarkably more expensive in terms of CPU time with respect to both GASPHOT and GALFIT, we limited the comparison to the case of simulated "blended" galaxies shown in Fig. 8.

For galaxies with $A_{\mathrm{thr}}>250$, the results of GIM2D are slightly worst than, but comparable to, those of both GALFIT and GASPHOT. The only important exception is a noticeable bias in the recovered magnitudes $(\sim 0.1 \mathrm{mag})$, which put more than $50 \%$ of all galaxies out of our "tolerance range". Also, the number of outliers found by GIM2D for the b/a and Sersic index parameters is higher than in the corresponding cases of GASPHOT and GALFIT (see Table 1). Finally, the quality (bias and scatter) of the fit provided by GIM2D shows a fast decrease at decreasing threshold area, rapidly leading to unacceptable results, in particular for the effective radius $R_{\mathrm{e}}$.

We notice that the results of this test cannot be compared with those shown in the original GIM2D paper (Marleau \& Simard 1998) and available on the web, because of the different approach used in the simulations: the original GIM2D simulation included two-component galaxies with a bulge following a de Vaucouleurs profile and keeping $n=4$ fixed during the fit. 


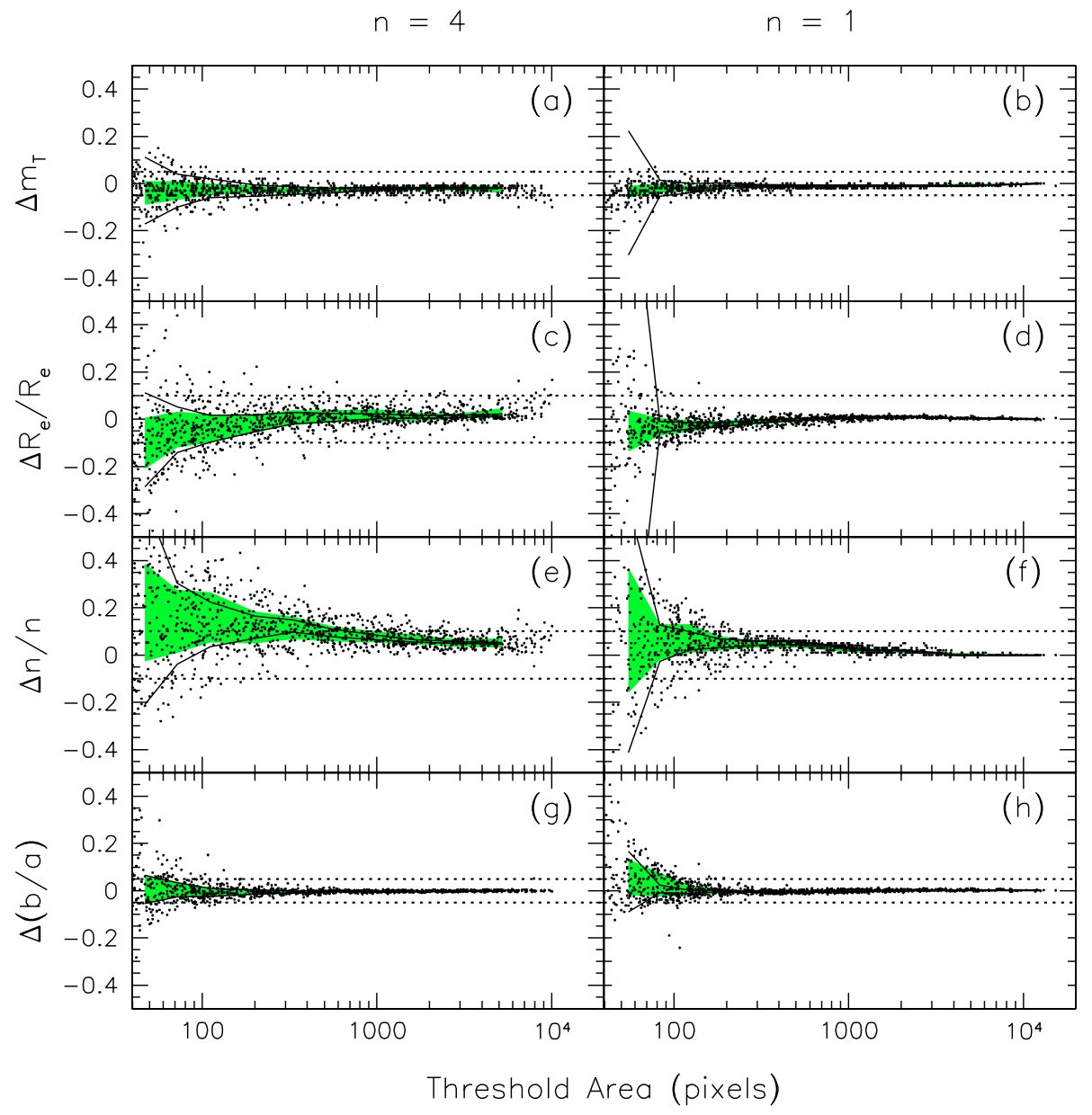

Fig. 11. Same as Fig. 5, but for GALFIT.
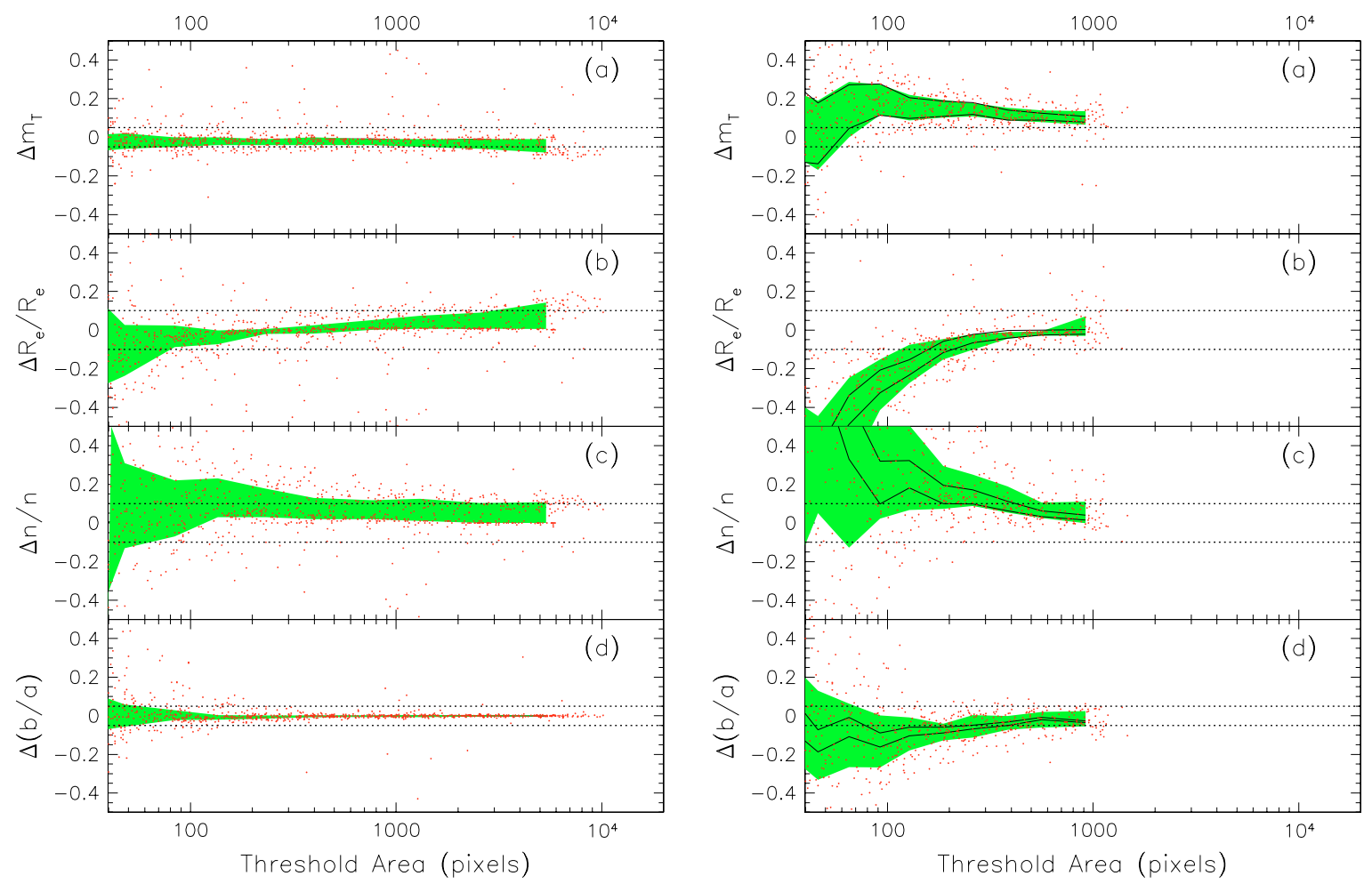

Fig. 12. Same as Fig. 8, but for GALFIT (left) and GIM2D (right). 


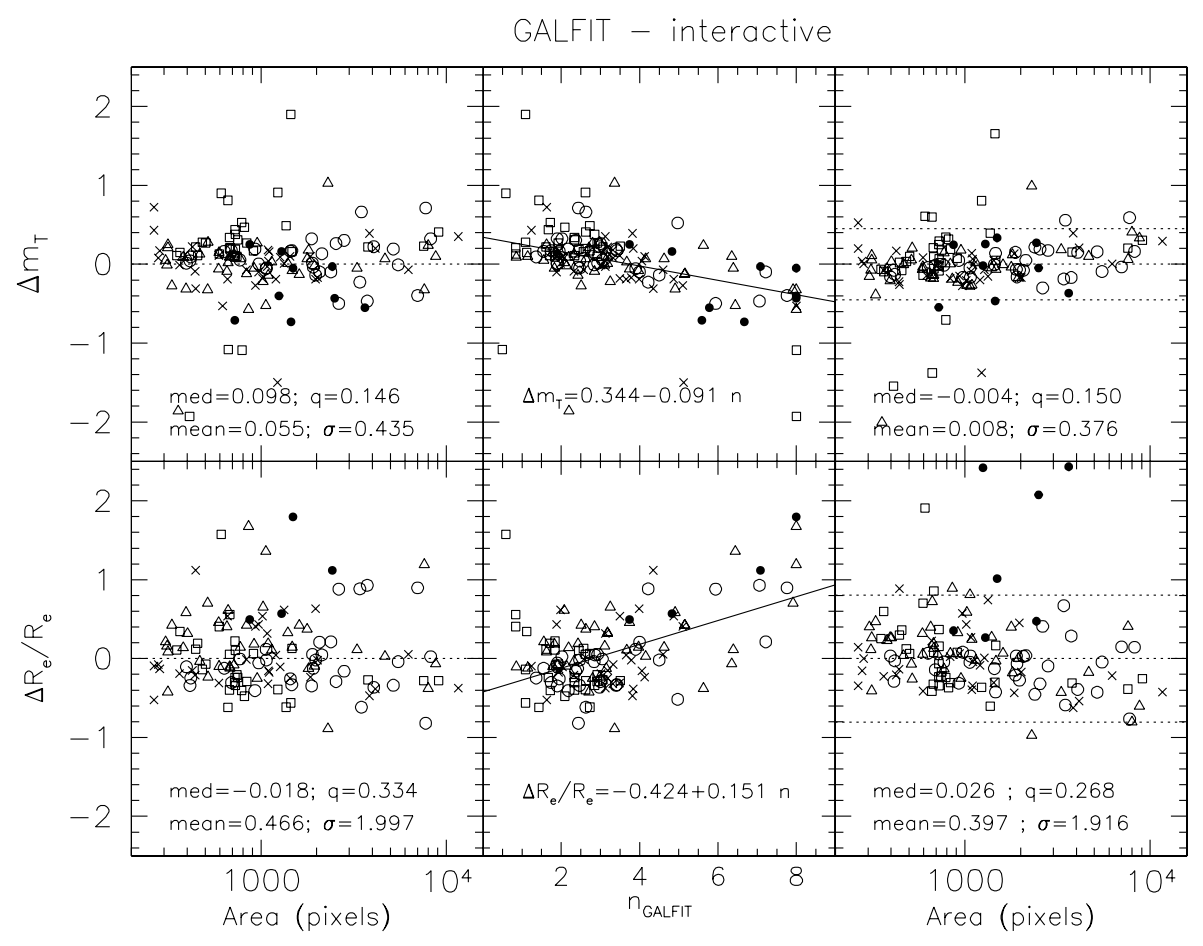

Fig. 13. Same as Fig. 9, but for GALFIT.

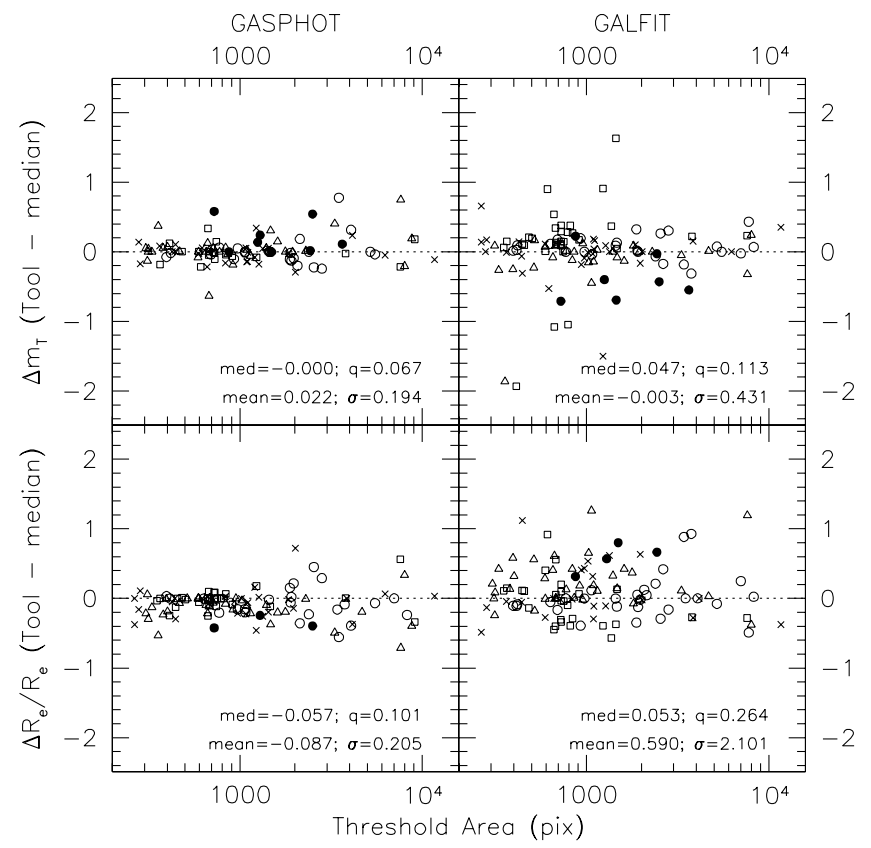

Fig. 14. Differences between magnitudes (upper panels) and radii (lower panels) obtained by GASPHOT (left panels) and GALFIT (right panels) for each object of the sample of real galaxies and the corresponding median values among GASPHOT, GALFIT and interactive photometry.

Here we fit instead single-component galaxies, but leave the value of the Sersic index free. Thus, we expect the results to be quite different in terms of bias and scatter.

In the above comparisons, the performances of both GALFIT and GIM2D were artificially lowered, since we forced them to model the galaxies with a pure Sersic law, while both tools were optimized to be used with multi-component models. On the other hand, using such models involves a large number of free parameters, thus making the tool extremely sensitive to the choice of the initial guesses of the parameters themselves. In the case of blind surface photometry of huge samples of real galaxies, we have no simple way to guess the initial values of $B / D$ and scale length ratios. Under these conditions, we feel that keeping reliable and physically meaningful results is likely to be very difficult.

\section{Conclusions}

In this paper we present an automatic tool, named GASPHOT, able to provide the relevant photometric and structural parameters for the thousands of objects typically involved in wide and/or deep field imaging. GASPHOT is heavily based on the use of SExtractor (Bertin \& Arnouts 1996), the most commonly used tool for the extraction of extended astronomical sources, which we are also using to obtain photometric and geometric profiles of all galaxies in the field. Such profiles are then fitted by a single Sersic law, convolved with a space-varying PSF extracted by the tool itself using the stars present in the frame. More precisely, we produced a simultaneous fitting of the light growth curves obtained along the major- and minor-axes of the galaxies. This approach exploits the robustness of the 1D fitting technique, saving at the same time the capability, typical of 2D approaches, of dealing with PSF convolution of flattened galaxies.

After a few steps of configuration, the tool automatically (without any interaction from the user) provides magnitude, axial ratio, scale radius, and Sersic index of the hundred - or thousand - galaxies in a wide/deep-field image. 
We devoted a good deal of effort to checking the robustness of the results when applying this tool to large samples of galaxies. In particular, we tested the performances of the tool on about 15000 simulated galaxies within wide ranges of magnitude, flattening, and radius. Such simulations included multicomponent galaxies and blended objects. Finally, we tested our tool on a set of real galaxies whose photometric parameters are evaluated interactively and are available in the literature.

Because our tool is intended to be used on hundreds of objects per run, going into detail for single objects is sacrificed for the sake of speed and robustness. We found that for objects with a threshold area greater than 250 pixels at a level of $2.5 \sigma_{\mathrm{bkg}}$, the photometric and structural parameters provided by GASPHOT lie within acceptable tolerance bands, even for composite $\left(R^{1 / 4}+\right.$ exp.) luminosity profiles, blended objects, and real-featured galaxies. A small amount of outliers are found, so that the results can be considered robust in a statistical sense.

We tested the results of GASPHOT against the widely used tools GALFIT (Peng et al. 2002) and GIM2D (Marleau \& Simard 1998). The comparison shows that the performances of GASPHOT are similar to those of GALFIT and GIM2D for large, regular (simulated) galaxies. Instead, the parameters retrieved by GASPHOT are much more stable for (real) galaxies having some kind of irregularity or blending, which is a crucial feature when dealing with blind surface photometry of huge galaxy samples.

Finally, the GASPHOT package is now available on the web: it can be downloaded at http://web.pd.astro.it/ pignatelli/GASPHOT. A short manual for the user is also available on the same site.

Acknowledgements. We wish to thank the anonymous referee for useful comments that greatly helped to improve the quality of the paper.

\section{References}

Abraham, R. G., Tanvir, N. R., Santiago, B. X., et al. 1996, MNRAS, 279, L47

Abraham, R. G., Valdes, F., Yee, H. K. C., \& van den Bergh, S. 1994, ApJ, 432, 75

Andreon, S., \& Cuillandre, J.-C. 2002, ApJ, 569, 144
Bertin, E., \& Arnouts, S. 1996, A\&AS, 117, 393

Blakeslee, J. P., Postman, M., Ford, H. C., et al. 2004, Amer. Astron. Soc. Meet., 204

Caon, N., Capaccioli, M., \& D’Onofrio, M. 1993, MNRAS, 265, 1013

Cassata, P., Cimatti, A., Franceschini, A., et al. 2005, MNRAS, 357, 903

Cimatti, A., Daddi, E., Cassata, P., et al. 2003, A\&A, 412, L1

Cimatti, A., Daddi, E., Renzini, A., et al. 2004, Nature, 430, 184

Ciotti, L. 1991, A\&A, 249, 99

Conselice, C. J. 2003, ApJS, 147, 1

Conselice, C. J., Bershady, M. A., \& Jangren, A. 2000, ApJ, 529, 886

D’Onofrio, M., Capaccioli, M., \& Caon, N. 1994, MNRAS, 271, 523

Durret, F., Adami, C., \& Lobo, C. 2002, A\&A, 393, 439

Fasano, G. 1994, Tech. Rep., Padova Observatory

Fasano, G., Bettoni, D., D’Onofrio, M., Kjærgaard, P., \& Moles, M. 2002, A\&A, 387, 26

Fasano, G., Falomo, R., \& Scarpa, R. 1996, MNRAS, 282, 40

Fasano, G., Poggianti, B., Bettoni, D., et al. 2003, Mem. Soc. Astron. It., 74,355

Fasano, G., Pignatelli, E., \& Cassata, P. 2005, in preparation

Franceschini, A., Silva, L., Fasano, G., et al. 1998, ApJ, 506, 600

Hudson, M. J., Davies, R. L., Lucey, J. R., et al. 2003, in Rev. Mex. Astron. Astrofis. Conf. Ser., 177

Jedrzejewski, R. I. 1987, MNRAS, 226, 747

Marleau, F. R., \& Simard, L. 1998, ApJ, 507, 585

McMahon, R. G., Walton, N. A., Irwin, M. J., et al. 2001, New Astronomy Review, 45, 97

Odewahn, S. C., Cohen, S. H., Windhorst, R. A., \& Philip, N. S. 2002, ApJ, 568, 539

Peng, C. Y., Ho, L. C., Impey, C. D., \& Rix, H. 2002, AJ, 124, 266

Pimbblet, K. A., \& Drinkwater, M. J. 2004, MNRAS, 347, 137

Pracy, M. B., Couch, W. J., Driver, S. P., \& Depropris, R. 2003, The Cosmic Cauldron, 25th meeting of the IAU, Joint Discussion 10, 18 July 2003, Sydney, Australia, 10

Ravindranath, S., Ferguson, H. C., Conselice, C., et al. 2004, ApJ, 604, L9

Rix, H., Barden, M., Beckwith, S. V. W., et al. 2004, ApJS, 152, 163

Sersic, J. L. 1968, Atlas de galaxias australes (Cordoba, Argentina: Observatorio Astronomico)

Simien, F., \& de Vaucouleurs, G. 1986, ApJ, 302, 564

Smail, I., Dressler, A., Couch, W. J., et al. 1997, ApJS, 110, 213

Takamiya, M. 1999, ApJS, 122, 109

Thompson, R. I. 2004, Amer. Astron. Soc. Meet., 204

Trujillo, I., Aguerri, J. A. L., Cepa, J., \& Gutiérrez, C. M. 2001, MNRAS, 321, 269

Ziegler, B. L., Saglia, R. P., Bender, R., et al. 1999, A\&A, 346, 13 